\title{
A new hierarchy of integrable systems associated to Hurwitz spaces
}

\author{
A. Kokotov* and D. Korotkin ${ }^{\dagger}$ \\ November 5, 2018 \\ Department of Mathematics and Statistics, Concordia University \\ 7141 Sherbrooke West, Montreal H4B 1R6, Quebec, Canada
}

\begin{abstract}
In this paper we introduce a new class of integrable systems, naturally associated to Hurwitz spaces (spaces of meromorphic functions over Riemann surfaces). The critical values of the meromorphic functions play the role of "times". Our systems give a natural generalization of the Ernst equation; in genus zero they realize the scheme of deformation of integrable systems proposed by Burtsev, Mikhailov and Zakharov. We show that any solution of these systems in rank 1 defines a flat diagonal metric (Darboux-Egoroff metric) together with a class of corresponding systems of hydrodynamic type and their solutions.
\end{abstract}

\section{Introduction}

Deformations of Riemann surfaces appear in different aspects of the theory of integrable systems. We mention the theory of systems of hydrodynamic type (in particular, the theory of Whitham deformations of integrable systems) [17, 5], theory of algebro-geometric solutions of equations with variable spectral parameter [14] (the main representative of this class is the Ernst equation from general relativity) and the theory of Frobenius manifolds 6, 7].

The Ernst equation

$$
\left((\xi-\bar{\xi}) G_{\xi} G^{-1}\right)_{\bar{\xi}}+\left((\xi-\bar{\xi}) G_{\bar{\xi}} G^{-1}\right)_{\xi}=0,
$$

where $G(\xi, \bar{\xi})$ is a matrix-valued function (the stationary axially symmetric Einstein equations are equivalent to this equation if $G$ is a $2 \times 2$ matrix with some additional symmetries) is the compatibility condition of the following linear system $(U-V$ pair $)$ 2, 22]:

$$
\frac{\partial \Psi}{\partial \xi}=\frac{G_{\xi} G^{-1}}{1-\nu} \Psi, \quad \frac{\partial \Psi}{\partial \bar{\xi}}=\frac{G_{\bar{\xi}} G^{-1}}{1+\nu} \Psi
$$

where $\nu$ is the following function of spectral parameter $l \in \mathbb{C}$ and variables $(\xi, \bar{\xi})$ :

$$
\nu=\frac{2}{\xi-\bar{\xi}}\left\{l-\frac{\xi+\bar{\xi}}{2}+\sqrt{(l-\xi)(l-\bar{\xi})}\right\} .
$$

*e-mail: alexey@mathstat.concordia.ca

†e-mail: korotkin@mathstat.concordia.ca 
Function $\nu(l)$ is nothing but the uniformization map of the genus zero Riemann surface which is the twofold covering of $l$-plane with two branch points at $l=\xi$ and $l=\bar{\xi}$. The map $l(\nu)$ is a rational map $\mathbb{C} P^{1} \rightarrow \mathbb{C} P^{1}$ of degree two with critical values $\xi$ and $\bar{\xi}$.

If the matrix dimension of $G$ is 1 , we can introduce the function $f=\ln G$ and the Ernst equation turns into the Euler-Darboux equation:

$$
\frac{\partial^{2} f}{\partial \xi \partial \bar{\xi}}+\frac{1}{2(\bar{\xi}-\xi)} \frac{\partial f}{\partial \xi}+\frac{1}{2(\xi-\bar{\xi})} \frac{\partial f}{\partial \bar{\xi}}=0 .
$$

The natural question is whether the Ernst equation is an isolated example of an integrable system related to the space of rational maps or it is possible to define natural analogs of the Ernst equation which would correspond to spaces of rational maps of arbitrary degree? More general, is it possible to go beyond the spaces of rational maps, and define natural analogs of the Ernst equation corresponding to general Hurwitz spaces $H_{g, N}$ of meromorphic functions of degree $N$ on Riemann surfaces of genus $g$ ? Is there any link between these higher analogs of Ernst equation and existing theories of systems of hydrodynamic type [17, 5], Frobenius manifolds and Darboux-Egoroff metrics corresponding to Hurwitz spaces [6]?

If we assume that $\nu$ in (1.1) is a constant, then the compatibility conditions of (1.1) leads to equation of principal chiral model; therefore, according to terminology proposed by Burtsev, Mikhailov and Zakharov 3, it is natural to call the Ernst equation the "deformation" of the principal chiral model equation. In [3] it was studied the general problem of deformation of a given integrable system which has $U-V$ pair, where matrices $U$ and $V$ are meromorphic functions of constant spectral parameter $\nu$. If one allows $\nu$ to depend on space variables $(x, y)$, then the zero curvature condition $U_{y}-V_{x}+[U, V]=0$ implies a set of differential equations for "variable spectral parameter" $\nu$ and poles of matrices $U$ and $V$. However, in [3] no regular method was given to solve these differential equations.

The first goal of this paper is to fill this gap. Namely, we show that the deformation scheme of [3] can be realized in terms of the spaces of rational maps $H_{0, N}$ of any given degree $N$. In this way we get a new hierarchy of non-autonomous nonlinear integrable systems. We show how solutions of the new systems can be described in terms of matrix Riemann-Hilbert problem and isomonodromic deformations. If the matrix dimension equals 1, these non-linear systems give rise to systems of linear non-autonomous second order partial differential equations, generalizing the Euler-Darboux equation.

Second, we extend our framework to construct a class of new integrable systems starting from an arbitrary Hurwitz space $H_{g, N}$ (space of meromorphic functions of degree $N$ on Riemann surfaces of genus $g$ ) for $g \geq 2$.

Third, in rank 1 (by rank of the integrable system we mean its matrix dimension) and any genus we observe a very close relationship between our systems, Darboux-Egoroff metrics, systems of hydrodynamic type and Frobenius manifolds. Moreover, our general formalism allows to give a simple description of systems of hydrodynamic type (as well as their solutions) associated to Hurwitz spaces $H_{g, N}$.

Let us describe our results in more details. Each rational map $R(\gamma)$ of degree $N$ defines an $N$-fold branch covering $\mathrm{E}$ of $\mathbb{C} P^{1}$; a point $P$ of $\mathrm{E}$ is a pair $(l, \gamma)$ such that

$$
l=R(\gamma)
$$

We assume that $R(\gamma)$ maps the infinity in $\gamma$-plane to the infinity in $l$-plane and $R(\gamma)=l+o(1)$ as 
$\gamma \rightarrow \infty$; then function $R(\gamma)$ has the form

$$
R(\gamma)=\gamma+\sum_{m=1}^{N-1} \frac{r_{m}}{\gamma-\mu_{m}}
$$

Denote the critical points of the map $R$ by $\gamma_{1}, \ldots, \gamma_{M}$; we shall assume that all of them are simple; then $M=2 N-2$ according to the Riemann-Hurwitz formula.

The ramification points of the covering $\mathrm{\iota}$ are denoted by $P_{m}=\left(l_{m}, \gamma_{m}\right)$ (they are simple since oll critical points of $R(\gamma)$ are simple); their projections $l_{m}$ on $l$-plane are called the branch points of the covering $\mathrm{E}$ (we adopt terminology of [9]); these are the values of the rational map $R(\gamma)$ at its critical points: $l_{m}=R\left(\gamma_{m}\right)$. In the sequel we shall assume that all $l_{m}$ are different.

Introduce two functions on the covering $\mathrm{E}$ : the function $\pi$, which projects $\mathrm{E}$ on $l$-plane: $\pi(P)=l$, and function $\nu$, which projects $\mathrm{E}$ to $\gamma$-plane: $\nu(P)=\gamma$. The map $\nu: \mathrm{E} \rightarrow \mathbb{C} P^{1}$ is a one-to-one map; its inverse is nothing but uniformization map of the covering $\mathrm{E}$. The maps $\nu$ and $\pi$ are related as follows: $R(\nu(P))=\pi(P)$.

Due to our assumption about the behaviour of $R(\gamma)$ at infinity, in a neighbourhood of the infinite point on some (we shall call it the first) sheet of $\mathrm{E}$ the map $\nu(P)$ behaves as follows: $\nu(P)=l+o(1)$.

The structure of Riemann surface on the branch covering $\mathrm{E}$ is defined as follows: in a neighbourhood of any point where $\mathrm{E}$ is non-ramified we can consider $l$ as local parameter. In a neighbourhood of a ramification point $P_{m}$ the local coordinate is chosen to be $x_{m}=\sqrt{l-l_{m}}$.

The branch covering $\mathrm{E}$ is completely defined by the branch points $l_{m}$ and a representation of fundamental group $\pi_{1}\left(\mathbb{C} P^{1} \backslash\left\{l_{1}, \ldots, l_{M}\right\}\right)$ in permutation group $S_{N}$. An element of permutation group describes permutation of sheets of the covering $\mathrm{E}$ if $l$ encircles a given contour in $\mathbb{C} P^{1} \backslash\left\{l_{1}, \ldots, l_{M}\right\}$. We shall consider local deformations of the covering $€$ such that this representation is kept fixed. Then the branch points $l_{m}$ can be considered as natural local coordinates on the space of rational maps; they will play the role of independent variables of our systems.

Let us fix some point $P_{0}$ of $\mathrm{E}$ such that its projection on $l$-plane $l_{0}=\pi\left(P_{0}\right)$ is independent of all $\left\{l_{m}\right\}$; let $\gamma_{0} \equiv \nu\left(P_{0}\right)$. Consider the following system of matrix linear differential equations for an auxiliary matrix-valued $r \times r$ function $\Psi\left(l,\left\{l_{m}\right\}\right)$ :

$$
\frac{\partial \Psi}{\partial l_{m}}=\frac{\gamma_{0}-\gamma_{m}}{\nu(P)-\gamma_{m}} J_{m} \Psi
$$

We assume that the solution $\Psi$ of (1.5) is normalized by the condition

$$
\Psi(l=\infty)=I
$$

If we put in (1.5) $P=P_{0}$, then the compatibility conditions of the system (1.5) imply that all $J_{m}$ are logarithmic derivatives of a matrix-valued function $G\left(\left\{l_{m}\right\}\right): J_{m}=G_{l_{m}} G^{-1}$. Besides that, the compatibility conditions of the system (1.5) imply the following coupled system of non-autonomous non-linear matrix partial differential equations of second order:

$$
\left(\left(\gamma_{0}-\gamma_{m}\right) G_{l_{m}} G^{-1}\right)_{l_{n}}=\left(\left(\gamma_{0}-\gamma_{n}\right) G_{l_{n}} G^{-1}\right)_{l_{m}}
$$

for all $m, n=1, \ldots, M$. We call the matrix dimension $r$ of the matrix $G$ the rank of the system (1.7).

For rational maps of degree two the system (1.7) coincides with the ordinary Ernst equation (1.1). If the rank equals 1, the systems (1.7) gives rise to certain generalization of the Euler-Darboux equation (1.2). 
We define the tau-function of these systems by the following system of compatible equations

$$
\frac{\partial}{\partial l_{m}} \ln \tau=\left.\frac{1}{2} \operatorname{res}\right|_{P_{m}} \operatorname{tr}\left\{\frac{\left(d_{P} \Psi \Psi^{-1}\right)^{2}}{d l}\right\},
$$

where $d_{P} \Psi=(\partial \Psi / \partial \nu(P)) d \nu(P)$; these equations fix $\tau$ up to an arbitrary constant multiplier if we assume that $\tau$ is holomorphic function of $\left\{l_{m}\right\}$.

Each system (1.7) possesses a subclass of "isomonodromic" solutions which can be built from an arbitrary solution of the Schlesinger system in the same matrix dimension. For this isomonodromic sector of solutions we link the tau-function to the Jimbo-Miwa tau-function of the Schlesinger system. This link generalizes the formula (found in [15]) relating the so-called "conformal factor" of the Ernst equation with Jimbo-Miwa tau-function. We also show how to construct solutions of the systems (1.7) from solutions of matrix Riemann-Hilbert problems on $\mathbb{C} P^{1}$.

After developing the theory of the systems (1.7), which are related to the space of rational maps $H_{0, N}$, we formulate a similar construction based on an arbitrary Hurwitz space $H_{g, N}$.

Consider in detail the rank 1 case. Let the $N$-fold branched covering $\mathrm{L}$ have genus $g$; then the number of branch points is equal to $M=2 g+2 N-2$ (as before, we assume that all the branch points are simple and have different projections on $l$-plane). Again, the projections $l_{m}$ of the branch points $P_{m}$ on $l$-plane can be used as local coordinates on $H_{g, N}$. Let us introduce some basis of canonical cycles on $\mathrm{E}$. Denote by $E(P, Q)$ the prime-form on $\mathrm{E}$, and by $B_{m}$ the normalized (all $a$-periods vanish) abelian differential of the second kind with the unique pole of second order at $P_{m}$ with leading coefficient 1. Let us choose two arbitrary points $P_{0}$ and $Q_{0}$ on $\mathrm{E}$ such that their projections $\pi\left(P_{0}\right)$ and $\pi\left(Q_{0}\right)$ on $l$-plane are $\left\{l_{m}\right\}$-independent. Then the $H_{g, N}$ analog of the genus zero systems (1.7) in rank 1 looks as follows:

$$
\frac{\partial^{2} f}{\partial l_{m} \partial l_{n}}-\frac{b\left(P_{m}, P_{n}\right)}{2}\left\{\frac{v_{n}}{v_{m}} \frac{\partial f}{\partial l_{m}}+\frac{v_{m}}{v_{n}} \frac{\partial f}{\partial l_{n}}\right\}=0, \quad m \neq n
$$

where

$$
b\left(P_{m}, P_{n}\right)=\left.\frac{B(P, Q)}{d x_{m}(P) d x_{n}(Q)}\right|_{P=P_{m}, Q=P_{n}}
$$

and $B(P, Q)=d_{P} d_{Q} \ln E(P, Q)$ is the Begmann kernel; $v_{m}=\int_{Q_{0}}^{P_{0}} B_{m}$.

Solutions of the system (1.9) can be constructed as follows. Let $l$ be an arbitrary closed contour on $\mathrm{E}$ such that its projection on the $l$-plane $\pi(l)$ is independent of $\left\{l_{m}\right\}$ and $P_{m} \notin l$ for any $m$. Let $h(P)$ be an arbitrary independent of $\left\{l_{m}\right\}$ Hölder-continuous function on $l$. Then the function

$$
f=\oint_{l} h(P) d_{P} \ln \frac{E\left(P_{0}, P\right)}{E\left(Q_{0}, P\right)}
$$

satisfies the system (1.9).

The systems (1.9) turn out to be in close relationship with diagonal flat metrics (Darboux-Egoroff metrics). Namely, consider the diagonal metric

$$
d s^{2}=\sum_{m=1}^{M} g_{m m} d l_{m}^{2},
$$

where $g_{m m}=\partial \ln \tau / \partial l_{m}$ and $\tau\left(\left\{l_{m}\right\}\right)$ is the tau-function corresponding to an arbitrary solution of (1.9). The rotation coefficients of this metric turn out to be given by the Bergmann kernel:

$$
\beta_{m n}=\frac{1}{2} b\left(P_{m}, P_{n}\right),
$$


i.e. they depend only on the covering $\mathrm{E}$ and don't depend on a particular solution of (1.9). In accordance with the Rauch variational formulas [8], which describe dependence of the Bergmann kernel on the branch points, these coefficients satisfy the equations

$$
\frac{\partial \beta_{m n}}{\partial l_{l}}=\beta_{m l} \beta_{l n}
$$

for distinct $l, m, n$, which, together with annihilation of all $\beta_{m n}$ by the operator $\sum_{k=1}^{M} \partial / \partial l_{k}$, guarantee flatness of the metric (1.11).

The Darboux-Egoroff metrics are known to be closely related to integrable classes of systems of hydrodynamic type, including, in particular, Whitham equations which describe slow deformations of the Riemann surfaces arising in dispersionless limit of finite-gap solutions of integrable systems. These systems are solvable via so-called generalized hodograph method [26, 17]. Our present scheme gives a short and simple formulation of the theory of the systems of hydrodynamic type corresponding to Hurwitz spaces.

We notice that the present paper has some overlap with the paper [19], devoted to isomonodromy deformations in higher genus, which appeared simultaneously with the first version of this text [13].

The paper is organized as follows. In section 2 we derive an auxiliary system of differential equations which describe dependence of the critical points of the rational map of the form (1.4) on the critical values. Then we show integrability of the systems (1.7), define corresponding tau-function, and discuss the relationship of these systems to the Riemann-Hilbert problem and the Schlesinger system. Here we also show how the systems (1.7) are related to deformations of integrable systems proposed in [3]. In section 3 we define analogs of the systems (1.7) related to Hurwitz spaces $H_{g, N}$ in arbitrary genus $g \geq 2$, and discuss their properties. In Sect.4 we show that each solution of these systems in rank 1 defines a Darboux-Egoroff metrics, and discuss related systems of hydrodynamic type in our framework, together with their solutions. Here we also outline the link between higher genus analogs of systems (1.7) and isomonodromic deformations on algebraic curves. In section 5 we discuss potential directions of future work.

\section{Non-autonomous integrable systems related to spaces of rational maps}

\subsection{Differential equations for critical points of rational maps}

Consider a rational map $R(\gamma)$ of degree $N$ of the form (1.4). If we choose the critical values $l_{1}, \ldots, l_{M}$ of the map (1.4) as independent parameters, each critical point $\gamma_{m}$ becomes a function of all $\left\{l_{m}\right\}$; the study of these functions is the main subject of this subsection. The function $\nu(P)$ depends on the variables $l_{1}, \ldots, l_{M}$ as parameters. In the sequel we shall denote the point of $\mathrm{E}$ which belongs to the $j$ th sheet and has a projection $l$ on the $l$-plane by $l^{(j)}$. The map $\nu(P)$ has its only pole at the point at infinity of some sheet of $\mathrm{E}$; we enumerate the sheets of $\mathrm{E}$ in such a way that this sheet has number one; therefore,

$$
\nu(P)=l+o(1) \quad \text { as } P \rightarrow \infty^{(1)} .
$$

Theorem 1 Function $\nu(P)$, considered locally as function of $l$ and depending on branch points $l_{1}, \ldots, l_{M}$ as parameters, satisfies the following equations:

$$
\frac{\partial \nu}{\partial l}=1+\sum_{n=1}^{M} \frac{\alpha_{n}}{\nu-\gamma_{n}}, \quad \frac{\partial \nu}{\partial l_{n}}=-\frac{\alpha_{n}}{\nu-\gamma_{n}} .
$$


where $\alpha_{m}$ are some functions of the branch points.

Proof. Consider the local behavior of the function $\nu(P)$ near a branch point:

$$
\nu(P)=\gamma_{m}+\kappa_{m} \sqrt{l-l_{m}}+O\left(l-l_{m}\right) \quad \text { as } \quad P \rightarrow P_{m}
$$

From (2.3) we conclude that

$$
\frac{\partial \nu}{\partial l}=\frac{\kappa_{m}}{2 \sqrt{l-l_{m}}}+O(1), \quad \frac{\partial \nu}{\partial l_{n}}=-\delta_{m n} \frac{\kappa_{m}}{2 \sqrt{l-l_{m}}}+O(1)
$$

as $P \rightarrow P_{m}$. By (2.3), we can rewrite these expansions using $\nu(P)$ as the global coordinate on $\mathrm{E}$ :

$$
\frac{\partial \nu}{\partial l}=\frac{\kappa_{m}^{2}}{2\left(\nu-\gamma_{m}\right)}+O(1), \quad \frac{\partial \nu}{\partial l_{n}}=-\delta_{m n} \frac{\kappa_{m}^{2}}{2\left(\nu-\gamma_{n}\right)}+O(1)
$$

where $\gamma_{n}=\nu\left(P_{n}\right)$. Moreover, from (2.1) we conclude that $\partial \nu / \partial l=1+o(1)$ and $\partial \nu / \partial l_{n}=o(1)$ as $\nu(P) \rightarrow \infty$. Therefore, $\partial \nu / \partial l$ is a meromorphic function of $\nu$ with simple poles at all the points $\gamma_{n}$ with residues $\kappa_{n}^{2} / 2$ and value 1 at infinity. Analogously, the function $\partial \nu / \partial l_{n}$ is a meromorphic function on $\mathbb{C} P^{1}$ with simple pole at $l_{n}$ and zero at infinity. Therefore, we get equations (2.2) with $\alpha_{n}=\kappa_{n}^{2} / 2$.

Corollary 1 The critical points $\gamma_{m}$ of the rational function (1.4) and residues $\alpha_{m}$ from (2.2) depend as follows on the critical values $l_{m}$ :

$$
\begin{gathered}
\frac{\partial \gamma_{m}}{\partial l_{n}}=\frac{\alpha_{n}}{\gamma_{n}-\gamma_{m}}, \quad m \neq n ; \quad \frac{\partial \gamma_{m}}{\partial l_{m}}=1+\sum_{n=1, n \neq m}^{M} \frac{\alpha_{n}}{\gamma_{m}-\gamma_{n}}, \\
\frac{\partial \alpha_{m}}{\partial l_{n}}=\frac{2 \alpha_{n} \alpha_{m}}{\left(\gamma_{n}-\gamma_{m}\right)^{2}}, \quad m \neq n ; \quad \frac{\partial \alpha_{m}}{\partial l_{m}}=-\sum_{n=1, n \neq m}^{M} \frac{2 \alpha_{n} \alpha_{m}}{\left(\gamma_{n}-\gamma_{m}\right)^{2}}
\end{gathered}
$$

for all $m, n=1, \ldots, M$.

Proof. Equations (2.5) and (2.6) follow from compatibility of equations (2.2).

Rational functions of the form (1.4) were introduced by Kupershmidt and Manin 21 in connection with Benney systems. The fact that the critical points of these functions satisfy equations (2.5),(2.6), follows from the recent paper by Gibbons and Tsarev [10 ${ }^{1}$. However, we did not find in existing literature the whole set of equations (2.2), (2.5), (2.6) associated to the functions (1.4).

Two-fold coverings. For an illustration consider the case $N=2$, when the covering $€$ has two sheets and two branch points $l_{1}, l_{2}$. Then the rational function $R(\gamma)$ (1.4) can be explicitly written in terms of its critical values $l_{1}$ and $l_{2}$ :

$$
R(\gamma)=\gamma+\frac{\left(l_{1}-l_{2}\right)^{2}}{16\left(\gamma-\frac{l_{1}+l_{2}}{2}\right)}
$$

\footnotetext{
${ }^{1}$ We thank E.Ferapontov, who attracted our attention to this work
} 
The map $\nu(P)$ looks as follows:

$$
\nu(P)=\frac{1}{2}\left(l+\frac{l_{1}+l_{2}}{2}+\sqrt{\left(l-l_{1}\right)\left(l-l_{2}\right)}\right) .
$$

The critical points $\gamma_{1,2}$ and variables $\alpha_{1,2}$ are given by:

$$
\begin{gathered}
\gamma_{1} \equiv \nu\left(l_{1}\right)=\frac{3 l_{1}+l_{2}}{4}, \quad \gamma_{2} \equiv \nu\left(l_{2}\right)=\frac{l_{1}+3 l_{2}}{4}, \\
\alpha_{1}=-\alpha_{2}=\frac{l_{1}-l_{2}}{8} .
\end{gathered}
$$

\subsection{Spaces of rational maps and new hierarchy of non-autonomous integrable systems}

Starting from an arbitrary branch $N$-fold covering $\mathrm{E}$ of genus zero we can construct a hierarchy of integrable systems as follows.

Fix some point $P_{0} \in \mathrm{E}$ such that its projection $l_{0}$ on $\mathbb{C} P^{1}$ is independent of all $\left\{l_{m}\right\}$, i.e. $\gamma_{0} \equiv \nu\left(P_{0}\right)$ depends on $\left\{l_{m}\right\}$ according to the equation

$$
R\left(\gamma_{0}\left(l_{1}, \ldots, l_{M}\right)\right)=l_{0} .
$$

Consider the following system of first order differential equations for a $r \times r$ matrix-valued function $\Psi\left(P,\left\{l_{m}\right\}\right)$ :

$$
\frac{d \Psi}{d l_{m}}=\frac{\gamma_{0}-\gamma_{m}}{\nu(P)-\gamma_{m}} J_{m} \Psi
$$

where $J_{m}$ are $r \times r$ matrix-valued functions of $\left\{l_{m}\right\}$.

As a corollary of compatibility conditions of the linear system (2.11) functions $J_{m}$ can be expressed in terms of the single function $G \equiv \Psi\left(P_{0}\right)$ :

$$
J_{m}=\frac{\partial G}{\partial l_{m}} G^{-1}
$$

moreover, the function $G$ satisfies the following system of non-linear partial differential equations:

$$
\left(\left(\gamma_{0}-\gamma_{m}\right) G_{l_{m}} G^{-1}\right)_{l_{n}}=\left(\left(\gamma_{0}-\gamma_{n}\right) G_{l_{n}} G^{-1}\right)_{l_{m}} .
$$

(to derive (2.13) from (2.11) one needs to make use of equations (2.2), (2.5) and (2.6).

We write the "total" derivative of $\Psi$ with respect to $l_{m}$ in (2.11) to emphasize that in (2.11) we consider $l$ and $l_{1}, \ldots, l_{m}$ as independent variables.

Alternatively, consider $\Psi(P)$ as a function of $\nu(P)$ and $l_{1}, \ldots, l_{M}$. Then, since $\nu(P)$ is itself a function of $l$ and $\left\{l_{m}\right\}$, we can rewrite (2.11) using the chain rule

$$
\frac{d \Psi}{d l_{m}}=\frac{\partial \Psi}{\partial l_{m}}+\frac{\partial \nu}{\partial l_{m}} \frac{\partial \Psi}{\partial \nu}
$$

where the notation $\partial \Psi / \partial l_{m}$ means that $\nu(P)$ remains fixed.

Then, using (2.2) for $\partial \nu / \partial l_{m}$, we rewrite (2.11) as follows:

$$
\frac{\partial \Psi(P)}{\partial l_{m}}-\frac{\alpha_{m}}{\nu(P)-\gamma_{m}} \frac{\partial \Psi(P)}{\partial \nu(P)}=\frac{\gamma_{0}-\gamma_{m}}{\nu(P)-\gamma_{m}} J_{m} \Psi(P) .
$$


A simple calculation making use of equations (2.13) and system (2.5), (2.6), shows that if $G\left(\left\{l_{m}\right\}\right)$ is a solution of non-linear system (2.13), then the 1-form

$$
\mathbf{q}=\sum_{m=1}^{M} \frac{\left(\gamma_{0}-\gamma_{m}\right)^{2}}{2 \alpha_{m}} \operatorname{tr}\left(G_{l_{m}} G^{-1}\right)^{2} d l_{m}
$$

is closed, $d \mathbf{q}=0$.

The closedness of the 1 -form $\mathbf{q}$ implies the existence of the potential $\tau$, which can naturally be called the tau-function of the non-linear system (2.13).

Definition 1 The function $\tau\left(l_{1}, \ldots, l_{M}\right)$, defined by the following system of equations:

$$
\frac{\partial \ln \tau}{\partial l_{m}}=\frac{\left(\gamma_{0}-\gamma_{m}\right)^{2}}{2 \alpha_{m}} \operatorname{tr}\left(G_{l_{m}} G^{-1}\right)^{2}
$$

up to an arbitrary constant multiplier, is called the tau-function of integrable system (2.13).

Using equations (2.13) (2.5), (2.6), we find that the second derivatives of the tau-function are given by the following expression:

$$
\frac{\partial^{2} \ln \tau}{\partial l_{m} \partial l_{n}}=\frac{\left(\gamma_{0}-\gamma_{m}\right)\left(\gamma_{0}-\gamma_{n}\right)}{2\left(\gamma_{m}-\gamma_{n}\right)^{2}} \operatorname{tr}\left\{G_{l_{m}} G^{-1} G_{l_{n}} G^{-1}\right\}
$$

for $m \neq n$.

Taking the residue of the linear system (2.14) at $P=P_{m}$, we find

$$
\alpha_{m} \Psi_{\gamma} \Psi^{-1}\left(P_{m}\right)=\left(\gamma_{0}-\gamma_{m}\right) G_{l_{m}} G^{-1}
$$

due to this relation the definition (2.16) allows an alternative formulation:

Definition 1' The tau-function of the system (2.13) is defined by the following equations:

$$
\frac{\partial \ln \tau}{\partial l_{m}}=\left.\frac{1}{2} \operatorname{res}\right|_{P_{m}}\left\{\frac{\operatorname{tr}\left(d_{P} \Psi \Psi^{-1}\right)^{2}}{d l}\right\}
$$

where

$$
d_{P} \Psi \Psi^{-1} \equiv \Psi_{\nu(P)} \Psi^{-1} d \nu(P)
$$

Let us prove the equivalence of the two definitions of the tau-function. Using $\nu(P)$ as a global coordinate on $\mathrm{E}$, we have:

$$
d l=\frac{\partial l}{\partial \nu} d \nu
$$

therefore,

$$
\frac{\operatorname{tr}\left\{\left(d_{P} \Psi \Psi^{-1}\right)^{2}\right\}}{d l}=\frac{\partial \nu}{\partial l} \operatorname{tr}\left\{\left(\Psi_{\nu} \Psi^{-1}\right)^{2}\right\} d \nu=\left\{1+\sum_{m=1}^{M} \frac{\alpha_{m}}{\nu-\gamma_{m}}\right\} \operatorname{tr}\left\{\left(\Psi_{\nu} \Psi^{-1}\right)^{2}\right\} d \nu,
$$

and (2.19) coincides with (2.16). 
The Ernst equation. For $N=2$ the hierarchy (2.13) reduces to a single equation. If one chooses point $P_{0}$ to coincide with $\infty^{(2)}$ [i.e. the point of $\mathrm{E}$ where $l=\infty$ and in a neighbourhood of which $\left.\sqrt{\left(l-l_{1}\right)\left(l-l_{2}\right)}=-l+\left(l_{1}+l_{2}\right) / 2+o(1)\right]$, we get

$$
\gamma_{0}=\nu\left(P_{0}\right)=\frac{l_{1}+l_{2}}{2}
$$

Taking into account expressions (2.9), we have

$$
\gamma_{0}-\gamma_{1}=\frac{l_{2}-l_{1}}{4}, \quad \gamma_{0}-\gamma_{2}=\frac{l_{1}-l_{2}}{4} .
$$

If we now assume that $l_{1}$ and $l_{2}$ are conjugated to each other: $l_{1}=\xi, l_{2}=\bar{\xi}$, then equation (2.13) takes the following form:

$$
\left((\xi-\bar{\xi}) G_{\xi} G^{-1}\right)_{\bar{\xi}}+\left((\xi-\bar{\xi}) G_{\bar{\xi}} G^{-1}\right)_{\xi}=0
$$

This equation is called the Ernst equation; it is equivalent to vacuum Einstein's equation for stationary axially symmetric spacetimes (the matrix $G$ in this case must be real, symmetric and must have unit determinant). In this case the linear system (2.11) is equivalent to the Lax representation of the Ernst equation found in 1978 by Belinskii-Zakharov [2] and Maison [22]. We notice that the Maison's Lax pair is a partial case of our linear system written in the form (2.11); whereas the Belinskii-Zakharov linear system is a partial case of (2.14).

Due to expressions (2.10) for $\alpha_{1,2}$, the definition of the tau-function $\tau$ can be written down as follows:

$$
\frac{\partial \ln \tau}{\partial \xi}=\frac{\xi-\bar{\xi}}{4} \operatorname{tr}\left(G_{\xi} G^{-1}\right)^{2}, \quad \frac{\partial \ln \tau}{\partial \bar{\xi}}=\frac{\bar{\xi}-\xi}{4} \operatorname{tr}\left(G_{\bar{\xi}} G^{-1}\right)^{2}
$$

Formula (2.23) coincides with the definition of the so-called conformal factor - one of the metric coefficients which correspond to a given solution of the Ernst equation.

\subsection{New integrable systems and deformation scheme of Burtzev-Mikhailov-Zakharov}

The possibility to construct the class of "deformed" integrable systems, or integrable systems with "variable spectral parameter", different from the Ernst equation, was first discovered by Burtzev, Mikhailov and Zakharov [3]. They proposed to consider the Lax pairs of the form

$$
\frac{\partial \Psi}{\partial x}=U \Psi, \quad \frac{\partial \Psi}{\partial y}=V \Psi
$$

where $x$ and $y$ are independent variables; matrices $U$ and $V$ depend on $(x, y)$ and the "variable spectral parameter" $\nu$ (which in turn depends on $(x, y)$ and the "hidden" spectral parameter $l$ ):

$$
\begin{aligned}
& U(x, y, \nu)=u_{0}(x, y)+\sum_{n=1}^{N_{1}} \frac{u_{n}(x, y)}{\nu(x, y)-\gamma_{n}(x, y)}, \\
& V(x, y, \nu)=v_{0}(x, y)+\sum_{n=1}^{N_{2}} \frac{v_{n}(x, y)}{\nu(x, y)-\tilde{\gamma}_{n}(x, y)} .
\end{aligned}
$$


As a part of compatibility conditions of the linear system (2.24), after an appropriate fractional-linear transformation in $\nu$-plane, the following system of equations for $\nu(x, y, l)$ must be satisfied:

$$
\frac{\partial \nu}{\partial y}+\sum_{m=1}^{N_{2}} \frac{b_{m}}{\nu-\tilde{\gamma}_{m}}=0, \quad \frac{\partial \nu}{\partial x}+\sum_{m=1}^{N_{1}} \frac{c_{m}}{\nu-\gamma_{m}}=0,
$$

where $b_{n}$ and $c_{n}$ are certain functions of $(x, y)$. The compatibility condition of the system (2.27) gives the following system for $\gamma_{n}(x, y)$ and $\tilde{\gamma}_{n}(x, y)$ :

$$
\begin{array}{rlrl}
\frac{\partial \gamma_{n}}{\partial y}+\sum_{m=1}^{N_{2}} \frac{b_{m}}{\gamma_{n}-\tilde{\gamma}_{m}} & =0, & \frac{\partial \tilde{\gamma}_{n}}{\partial x}+\sum_{m=1}^{N_{1}} \frac{c_{m}}{\tilde{\gamma}_{n}-\gamma_{m}}=0, \\
\frac{\partial c_{n}}{\partial y}-2 c_{n} \sum_{m=1}^{N_{2}} \frac{b_{m}}{\left(\gamma_{n}-\tilde{\gamma}_{m}\right)^{2}}=0, & \frac{\partial b_{n}}{\partial x}-2 b_{n} \sum_{m=1}^{N_{1}} \frac{c_{m}}{\left(\tilde{\gamma}_{n}-\gamma_{m}\right)^{2}}=0 .
\end{array}
$$

It is easy to establish the relationship between solutions of the system (2.27), (2.28), (2.29), and solutions of our system (2.2), (2.5), (2.6).

Namely, suppose that function $\nu\left(l,\left\{l_{m}\right\}_{m=1}^{M}\right)$ satisfies equations (2.2) with respect to variables $l_{m}$. Assume that $M=N_{1}+N_{2}$ and split the set of variables $\left\{l_{1}, \ldots, l_{N_{1}+N_{2}}\right\}$ into two subsets: $\left\{l_{1}, \ldots, l_{N_{1}}\right\}$ and $\left\{\tilde{l}_{1}, \ldots, \tilde{l}_{N_{2}}\right\}$ where $\tilde{l}_{n} \equiv l_{N_{1}+n}, n=1, \ldots, N_{2}$. In the same way we split the set $\left\{\gamma_{m}\right\}$ of values of function $\nu(P)$ at these points:

$$
\left\{\gamma_{1}, \ldots, \gamma_{M}\right\}=\left\{\gamma_{1}, \ldots, \gamma_{N_{1}}\right\} \cup\left\{\tilde{\gamma}_{1}, \ldots, \tilde{\gamma}_{N_{2}}\right\},
$$

where $\tilde{\gamma}_{n} \equiv \gamma_{N_{1}+n}, n=1, \ldots, N_{2}$.

Now assume that the "untilded" variables $l_{1}, \ldots, l_{N_{1}}$ are arbitrary functions of variable $x$ and the "tilded" variables $\tilde{l}_{1}, \ldots, \tilde{l}_{N_{2}}$ are arbitrary functions of variable $y$. Then using (2.2) we get the derivative of $\nu(P)$ with respect to $x$ :

$$
\frac{\partial \nu}{\partial x}=\sum_{m=1}^{N_{1}} \frac{\partial \nu}{\partial l_{m}} \frac{\partial l_{m}}{\partial x}=-\sum_{m=1}^{N_{1}} \frac{\partial l_{m}}{\partial x} \frac{\alpha_{m}}{\nu-\gamma_{m}} ;
$$

therefore,

$$
\frac{\partial \nu}{\partial x}+\sum_{m=1}^{N_{1}} \frac{c_{m}}{\nu-\gamma_{m}}=0
$$

where $c_{m} \equiv \alpha_{m} \frac{\partial l_{m}}{\partial x}$; this coincides with the second equation in (2.27). The first equation in (2.27) is obtained in the same way after identification

$$
b_{m} \equiv \alpha_{N_{1}+m} \frac{\partial l_{N_{1}+m}}{\partial y} .
$$

Equations (2.28) and (2.29) for $\gamma_{n}, b_{n}$ and $c_{n}$ as functions of $(x, y)$ arise as compatibility conditions of equations for $\nu_{x}$ and $\nu_{y}$.

Therefore, spaces of rational maps of given degree provide solutions of the system (2.27), (2.28), (2.29) if

1. We split the set of the branch points $\left\{l_{m}\right\}$ into two subsets and

2. Assume that one subset contains the branch points which are arbitrary functions of $x$ only and another subset contains the branch points which are arbitrary functions of $y$ only.

Concluding, we see that the system (2.13) provides a realizations of the deformation scheme of [3]. 


\subsection{Solutions via the Riemann-Hilbert problem}

Solutions of systems (2.13) in terms of matrix Riemann-Hilbert problem are given by the following theorem.

Theorem 2 Consider a closed contour $l$ on the branch covering $E$ such that its projection $\pi(l)$ on the $l$-plane is independent of $\left\{l_{m}\right\}$. Assume that none of the ramification points $P_{m}$ belongs to $l$. Define a non-degenerate matrix function $l \ni P \mapsto H(P)$ which is independent of $\left\{l_{m}\right\}$. Suppose that a function $\Psi(P)$ satisfies the following Riemann-Hilbert problem on $E$ :

1. $\Psi(P)$ is holomorphic and non-degenerate on $E$ outside of the contour $l$ where it has the finite boundary values related as follows:

$$
\Psi_{+}(P)=\Psi_{-}(P) H(P)
$$

2. $\Psi$ satisfies the normalization condition at $\infty^{(1)}$ (this is the point of $E$ such that $\pi\left(\infty^{(1)}\right)=\infty$ and $\left.\nu\left(\infty^{(1)}\right)=\infty\right)$ :

$$
\Psi\left(\infty^{(1)}\right)=I
$$

Then the function $\Psi$ satisfies the linear system (2.11) with

$$
G=\Psi\left(P_{0}\right),
$$

and, therefore, the function $G$ solves the system (2.13).

Proof. Let $\Psi$ satisfy the Riemann-Hilbert problem. Consider its logarithmic derivative $\Psi_{l_{m}} \Psi^{-1}$. Due to the independence of the contour $l$ and the matrix $H(P)$ of $l_{m}$, this logarithmic derivative is single-valued on . Moreover, it is obviously holomorphic on $\mathrm{E}$ outside of the point $P_{m}$. Let us write the first two terms of the Taylor expansion of $\Psi(P)$ near $P_{m}$ :

$$
\Psi=\Psi_{0}+\sqrt{l-l_{m}} \Psi_{1}+\ldots
$$

then

$$
\Psi_{l_{m}} \Psi^{-1}=\frac{-1}{2 \sqrt{l-l_{m}}} \Psi_{1} \Psi_{0}^{-1}+O(1)
$$

Therefore, in terms of the uniformization map $\nu(P)$, we can write

$$
\Psi_{l_{m}} \Psi^{-1}=\frac{C_{m}}{\nu(P)-\gamma_{m}}
$$

with some matrix $C_{m}$ which is independent of $P$. The constant term in this formula is absent due to normalization condition (2.33). To compute $C_{m}$ we put $P=P_{0}$, i.e. $\nu(P)=\gamma_{0}$; then, using (2.34), we get

$$
C_{m}=\left(\gamma_{0}-\gamma_{m}\right) G_{l_{m}} G^{-1},
$$

which shows that the function $\Psi$, indeed, satisfies (2.11), and the corresponding function $G$ solves the system (2.13).

Below we also establish a relationship between systems (2.13) and another type of the RiemannHilbert problems, where the function $\Psi$ is allowed to have regular singularities. 


\subsection{Relationship to isomonodromic deformations}

The set of solutions of each system (2.13) has a subset of solutions corresponding to isomonodromic deformations of ordinary differential equations with meromorphic matrix coefficients:

$$
\frac{d \Psi}{d \gamma}=A(\gamma) \Psi, \quad A(\gamma)=\sum_{j=1}^{L} \frac{A_{j}}{\gamma-z_{j}}
$$

where $A_{j} \in g l(r)$ are certain matrices which are independent of $\gamma$ and such that $\sum_{j=1}^{L} A_{j}=0$. Consider $G L(r)$-valued solution $\Psi(\gamma)$ of (2.35) satisfying the initial condition at some point $\gamma_{0} \in \mathbb{C} P^{1}$ :

$$
\Psi\left(\gamma_{0}\right)=I
$$

The solution $\Psi(\gamma)$ has regular singularities at the points $z_{j}$; this function is generically non-singlevalued in the $\gamma$-plane: it gains the right multipliers $M_{j}$ under analytical continuation along contours starting at $\gamma_{0}$ and encircling poles $z_{j}$. The matrices $M_{j}$ are called the monodromy matrices of equation (2.35); they generate the monodromy group of equation (2.35). If all the monodromy matrices are independent of the positions of singularities $\left\{z_{j}\right\}$, then (in the generic case, when none of the eigenvalues of each matrix $A_{j}$ differ by an integer number) the function $\Psi$ satisfies the following equations with respect to the positions of singularities $z_{j}$ :

$$
\frac{d \Psi}{d z_{j}}=\left(\frac{A_{j}}{\gamma_{0}-z_{j}}-\frac{A_{j}}{\gamma-z_{j}}\right) \Psi .
$$

Compatibility of equations (2.35) and (2.37) is equivalent to the Schlesinger system for the functions $A_{j}\left(\left\{z_{k}\right\}\right)$ :

$$
\begin{aligned}
& \frac{\partial A_{j}}{\partial z_{k}}=\frac{\left[A_{j}, A_{k}\right]}{z_{j}-z_{k}}-\frac{\left[A_{j}, A_{k}\right]}{\gamma_{0}-z_{k}}, \quad j \neq k ; \\
& \frac{\partial A_{j}}{\partial z_{j}}=-\sum_{k \neq j}\left(\frac{\left[A_{k}, A_{j}\right]}{z_{k}-z_{j}}-\frac{\left[A_{k}, A_{j}\right]}{z_{k}-\gamma_{0}}\right) .
\end{aligned}
$$

The tau-function $\tau_{J M}$ of the Schlesinger system, introduced by Jimbo, Miwa and their collaborators 12, is defined by the following system:

$$
\frac{\partial}{\partial z_{j}} \ln \tau_{J M}=\left.\frac{1}{2} \operatorname{res}\right|_{\gamma=z_{j}} \operatorname{tr}\left(\Psi_{\gamma} \Psi^{-1}\right)^{2} ; \quad \frac{\partial \tau_{J M}}{\partial \bar{z}_{j}}=0 .
$$

Each solution of the Schlesinger system induces a solution of hierarchy (2.13) according to the following theorem:

Theorem 3 Consider a solution $\left\{A_{j}\left(\left\{z_{k}\right\}\right)\right\}$ of the Schlesinger system (2.38), together with the taufunction $\tau_{J M}$ and the corresponding solution $\Psi\left(\gamma,\left\{z_{j}\right\}\right)$ of the linear system (2.35), 2.37) normalized by (2.36). Let $E$ be a genus 0 covering of the l-plane with simple ramification points $P_{1}, \ldots, P_{M}$; choose the map $\nu(P)$ to satisfy (2.1). Consider an arbitrary set of points $P_{0}$ and $Q_{1}, \ldots, Q_{L}$ on $E$ such that their projections $l_{0}$ and $\mu_{1}, \ldots, \mu_{L}$ on $\mathbb{C} P^{1}$ (respectively) are independent of the branch points $l_{1}, \ldots, l_{M}$. Let us assume that the arguments of the solution and the tau-function of the Schlesinger system are given by the formulas

$$
z_{j}=\nu\left(Q_{j}\right), \quad \gamma_{0}=\nu\left(P_{0}\right)
$$


Then the function

$$
\Psi\left(P,\left\{l_{m}\right\}\right)=\left.\Psi\left(\gamma, \gamma_{0},\left\{z_{j}\right\}\right)\right|_{\gamma=\nu(P), \gamma_{0}=\nu\left(P_{0}\right), z_{j}=\nu\left(Q_{j}\right)}
$$

satisfies the linear system (2.11) of the hierarchy (2.13) with the functions $J_{m}$ defined by

$$
J_{m}\left(\left\{l_{n}\right\}\right)=\frac{\alpha_{m}}{\gamma_{m}-\gamma_{0}} A\left(\gamma_{m}\right) .
$$

Therefore, according to (2.12), we have $J_{m}=G_{l_{m}} G^{-1}$ for some function $G\left(\left\{l_{m}\right\}\right)$, which satisfies the system (2.13).

The corresponding tau-function $\tau$ is related to the Jimbo-Miwa tau-function as follows:

$$
\tau\left(\left\{l_{m}\right\}\right)=\left.\prod_{j=1}^{L}\left(\frac{\partial \nu}{\partial l}\left(Q_{j}\right)\right)^{\operatorname{tr} A_{j}^{2} / 2} \tau_{J M}\left(\left\{z_{j}\right\}\right)\right|_{z_{j}=\nu\left(Q_{j}\right)},
$$

where the derivative $\partial \nu / \partial l$ is given by equation (2.2).

Proof. Taking the derivative of the function $\Psi(P)$ with respect to $l_{m}$ using the chain rule, we get

$$
\begin{gathered}
\frac{\partial \Psi(P)}{\partial l_{m}}=\frac{\partial \Psi}{\partial \nu(P)} \frac{\partial \nu(P)}{\partial l_{m}} \\
+\sum_{j=1}^{L} \frac{\partial \Psi}{\partial z_{j}} \frac{\partial z_{j}}{\partial l_{m}}=\sum_{j=1}^{L} \frac{A_{j}}{\nu(P)-z_{j}} \frac{\alpha_{m}}{\gamma_{m}-\nu(P)} \Psi-\sum_{j=1}^{L} \frac{A_{j}}{\nu(P)-z_{j}} \frac{\alpha_{m}}{\gamma_{m}-z_{j}} \Psi \\
=-\frac{\alpha_{m}}{\nu(P)-\gamma_{m}} \sum_{j=1}^{L} \frac{A_{j}}{z_{j}-\gamma_{m}} \Psi=\frac{\gamma_{0}-\gamma_{m}}{\nu(P)-\gamma_{m}} J_{m} \Psi
\end{gathered}
$$

where functions $J_{m}$ are defined by (2.42).

Let us show how to prove the relation (2.43) between tau-functions. Taking into account the definition of Jimbo-Miwa tau-functions (2.39), we have:

$$
\frac{1}{2} \operatorname{tr}\left(\Psi_{\gamma} \Psi^{-1}\right)^{2}=\sum_{j=1}^{L} \frac{\operatorname{tr} A_{j}^{2}}{2\left(\gamma-z_{j}\right)^{2}}+\sum_{j=1}^{L} \frac{\partial_{z_{j}} \ln \tau_{J M}}{\gamma-z_{j}}
$$

Now, using definition (2.16) of the tau-function $\tau$, we get

$$
\frac{\partial \ln \tau}{\partial l_{m}}=\left.\frac{\alpha_{m}}{2} \operatorname{tr}\left(\Psi_{\nu} \Psi^{-1}\right)^{2}\right|_{P=P_{m}}=\sum_{j=1}^{L} \frac{\operatorname{tr} A_{j}^{2}}{2} \frac{\alpha_{m}}{\left(\gamma_{m}-z_{j}\right)^{2}}+\sum_{j=1}^{L} \frac{\alpha_{m}}{\gamma_{m}-z_{j}} \partial_{z_{j}} \ln \tau_{J M} .
$$

By (2.2), we see that

$$
\frac{\partial}{\partial l_{m}} \ln \left\{\frac{\partial \nu}{\partial l}\left(Q_{j}\right)\right\}=\frac{\alpha_{m}}{\left(\gamma_{m}-z_{j}\right)^{2}}, \quad \frac{\partial z_{j}}{\partial l_{m}}=\frac{\alpha_{m}}{\gamma_{m}-z_{j}}
$$

therefore, applying the chain rule in (2.44) (and taking into account that $\operatorname{tr} A_{j}^{2}$ are integrals of the Schlesinger system), we come to (2.43).

Remark 1 The relationship between the systems (2.13) and isomonodromic deformations is a generalization of the link between the Ernst equation and the Schlesinger system established in [15]. 


\subsection{Rank 1 systems}

When the function $G$ from (2.13) is a scalar one, the system (2.13) can be rewritten as a system of linear scalar second order differential equations in terms of the function $f\left(\left\{l_{m}\right\}\right)=\ln G$ :

$$
\left(\gamma_{m}-\gamma_{n}\right)^{2} \frac{\partial^{2} f}{\partial l_{m} \partial l_{n}}-\alpha_{n} \frac{\gamma_{m}-\gamma_{0}}{\gamma_{n}-\gamma_{0}} \frac{\partial f}{\partial l_{m}}-\alpha_{m} \frac{\gamma_{n}-\gamma_{0}}{\gamma_{m}-\gamma_{0}} \frac{\partial f}{\partial l_{n}}=0, \quad m \neq n .
$$

In derivation of the system (2.45) from (2.13) we used equations (2.5).

In particular, any solution of the matrix system (2.13) gives a solution of the scalar system (2.45) if we put $f=\ln \operatorname{det} G$.

The linear system (2.11) turns in rank 1 into the scalar system

$$
\frac{\partial \psi(P)}{\partial l_{m}}=\frac{\gamma_{0}-\gamma_{m}}{\nu(P)-\gamma_{m}} \frac{\partial f}{\partial l_{m}}
$$

where $\psi\left(P,\left\{l_{m}\right\}\right)=\ln \Psi$. As well as in the matrix case, the function $\psi$ is generically non-single-valued on $\mathrm{E}$.

The definition of tau-function (2.16) now looks as follows:

$$
\frac{\partial \ln \tau}{\partial l_{m}}=\frac{\left(\gamma_{0}-\gamma_{m}\right)^{2}}{2 \alpha_{m}}\left\{\frac{\partial f}{\partial l_{m}}\right\}^{2} .
$$

Alternatively, it can be rewritten in terms of the function $\psi(P)$, using (2.19):

$$
\frac{\partial \ln \tau}{\partial l_{m}}=\left.\frac{1}{2} \operatorname{res}\right|_{P_{m}}\left\{\frac{(d \psi)^{2}}{d l}\right\}
$$

where $d \psi(P)=\psi_{\nu}(P) d \nu(P)$.

Let us discuss the solutions of system (2.46) and equation (2.45).

Theorem 4 Let $l$ be an arbitrary smooth closed contour on $E$ such that its projection on the l-plane $\pi(l)$ is independent of $\left\{l_{m}\right\}$ and $P_{m} \notin l$ for any $m$. Consider on $l$ an arbitrary Hölder-continuous function $h(P)$ independent of $\left\{l_{m}\right\}$. Then the function

$$
f=\oint_{l} \frac{h(Q) d \nu(Q)}{\nu(Q)-\gamma_{0}}
$$

satisfies system 2.45).

Proof. In the scalar case we know explicitly the solution of an arbitrary Riemann-Hilbert problem from theorem 2. If we define the function

$$
h(P)=\frac{1}{2 \pi i} \ln H(P)
$$

(we assume that it is single-valued on $l$ i.e. the index of $H(P)$ on $l$ equals 0 ), the Riemann-Hilbert problem (2.32) becomes additive:

$$
\psi_{+}(P)=\psi_{-}(P)+2 \pi i h(P)
$$


for $P \in l$ (we recall that $\psi=\ln \Psi$ ); here $\psi_{ \pm}$stands for the boundary values of the function $\psi$ on $l$. Normalization condition (2.33) turns into

$$
\psi\left(\infty^{(1)}\right)=0
$$

The solution of (2.50), (2.51) for a Hölder-continuous functions $h(P)$ is given by the Cauchy integral

$$
\psi(P)=\oint_{l} \frac{h(Q) d \nu(Q)}{\nu(Q)-\nu(P)},
$$

which implies (2.49) at $P=P_{0}$, when $\nu(P)=\gamma_{0}$.

Formula (2.49) can also be verified as follows. First, one can check by the direct substitution, using equations (2.2), (2.5), (2.6), that the function

$$
f=\frac{1}{\nu(Q)-\gamma_{0}} \frac{\partial \nu(Q)}{\partial l}
$$

satisfies system (2.45) for any $l \equiv \pi(Q)$ independent of $\left\{l_{m}\right\}$. Using linearity of equation (2.45) we can consider the superposition of these solutions at different $Q \in l$ with an arbitrary $\left\{l_{m}\right\}$-independent measure $h(Q)$, which leads to (2.49).

In fact, we can consider any (say, compact) subset $D \subset \mathrm{E}$ whose projection on $l$-plane is $\left\{l_{m}\right\}$ independent, and such that $P_{m} \notin D$. We can define an arbitrary $\left\{l_{m}\right\}$-independent measure $d \mu(Q)$ on $D$; then the superposition principle implies that the function

$$
f=\int_{D} \frac{h(Q)}{\nu(Q)-\gamma_{0}} \frac{\partial \nu(Q)}{\partial l} d \mu(Q)
$$

is a solution of (2.45).

The Euler-Darboux equation. As we noticed earlier, for the twofold covering with two branch points $l_{1}=\xi$ and $l_{2}=\bar{\xi}$ the system (2.13) is equivalent to Ernst equation (2.22). In scalar case we get the following equation in terms of $f=\ln G$ :

$$
f_{\xi \bar{\xi}}-\frac{f_{\xi}-f_{\bar{\xi}}}{2(\xi-\bar{\xi})}=0 .
$$

If we introduce the real coordinates $(z, \rho)$ such that $\xi=z+i \rho, \bar{\xi}=z-i \rho$, this equation takes the form of Euler-Darboux equation:

$$
f_{z z}+\frac{1}{\rho} f_{\rho}+f_{\rho \rho}=0 .
$$

From (2.8), taking into account that $P_{0}=\infty^{(2)}$ and $\gamma_{0}=\left(l_{1}+l_{2}\right) / 2$, we obtain:

$$
\frac{1}{\nu(P)-\gamma_{0}} \frac{\partial \nu(P)}{\partial l}=\frac{1}{[(l-\xi)(l-\bar{\xi})]^{1 / 2}}
$$

Then representation (2.49) gives the solution [4] of the Euler-Darboux equation:

$$
f=\oint_{l} \frac{h(l) d l}{[(l-\xi)(l-\bar{\xi})]^{1 / 2}} .
$$




\section{Generalization to higher genus}

Here we discuss possible ways to define systems, analogous to (2.13), starting from Hurwitz spaces $H_{g, N}$ (which are spaces of meromorphic functions of degree $N$ over Riemann surfaces of genus $g$ ) in genus $g \geq 2$ (we skip the case $g=1$ when the classification of stable bundles is rather special [1], see also remark 2 below).

As before, denote the critical points of a meromorphic function $\pi(P)$ on a Riemann surface of genus $g$ by $P_{1}, \ldots, P_{M}$ and their images in $\mathbb{C} P^{1}$ by $l_{m}=\pi\left(P_{m}\right)$. This meromorphic function realizes the Riemann surface as a ramified $N$-fold covering $\mathrm{E}$ of genus $g$ of the $l$-sphere with ramification points $P_{1}, \ldots, P_{M}$. Assuming that all the branch points $l_{m}$ are different and simple, we get, according to the Riemann-Hurwitz formula:

$$
M=2 g+2 N-2 .
$$

Let's introduce on $\mathrm{E}$ a canonical basis of cycles $\left(a_{\alpha}, b_{\alpha}\right)(\alpha=1, \ldots, g)$ and corresponding basis of holomorphic 1-forms $w_{\alpha}(P)(\alpha=1, \ldots, g)$, normalized by $\oint_{a_{\alpha}} w_{\beta}=\delta_{\alpha \beta}$.

\subsection{Rank 1 systems in arbitrary genus}

The scalar systems (2.45) admit natural generalization to the Hurwitz spaces $H_{g, N}$. Denote the primeform on $\mathrm{E}$ by $E(P, Q)$ (where $P, Q \in \mathrm{E}$ ); introduce the Bergmann kernel $B(P, Q)=d_{P} d_{Q} \ln E(P, Q)$. By $B_{m}(P)$ we denote the meromorphic differential of 2 nd kind with vanishing $a$-periods and single pole at $P_{m}$ of the second order with the following local behaviour:

$$
B_{m}(P)=\left(\frac{1}{x_{m}^{2}}+O(1)\right) d x_{m}
$$

as $P \rightarrow P_{m}$, where $x_{m}=\sqrt{l-l_{m}}$ is a local parameter near $P_{m}$. Differentials $B_{m}$ are related to the Bergmann kernel as follows:

$$
B_{m}(P)=\left.\frac{B(P, Q)}{d x_{m}(Q)}\right|_{Q=P_{m}}
$$

Corresponding abelian integrals we denote by $\varnothing_{m}$ :

$$
\emptyset_{m}(P)=\left.\int_{Q_{0}}^{P} B_{m} \equiv \frac{d}{d x_{m}(Q)}\left\{\ln \frac{E(P, Q)}{E\left(Q_{0}, Q\right)}\right\}\right|_{Q=P_{m}}
$$

where $Q_{0} \in \mathrm{E}$ is a base-point such that its projection on $l$-plane does not depend on $\left\{l_{n}\right\}$.

Let us prove the following variational formula.

Theorem 5 Assume that the local parameters $x_{P}, x_{Q}$ don't depend on (some) $l_{m}$. Introduce the symmetric function

$$
b(P, Q)=\frac{B(P, Q)}{d x_{P} d x_{Q}} \equiv \frac{\partial^{2}}{\partial x_{P} \partial x_{Q}} \ln E(P, Q) .
$$

Then dependence of $b(P, Q)$ on the branch point $l_{m}$ is given by the following equation:

$$
\frac{\partial b(P, Q)}{\partial l_{m}}=\frac{1}{2} b\left(P, P_{m}\right) b\left(Q, P_{m}\right)
$$


Proof. Formula (3.4) is closely related to the Rauch variational formulas [25, 8] which describe dependence of holomorphic differentials on the branch points. The proof is also very similar. Namely, consider the $l_{m}$-derivative of the Bergmann kernel: $\partial B(P, Q) / \partial l_{m}$. This is a symmetric 1 -form on $\mathrm{E} \times \mathrm{\textrm {E }}$. Consider the Taylor series of $B(P, Q)$ with respect to its first argument $P$ in a neighbourhood of ramification point $P_{m}$ :

$$
B(P, Q)=\left\{a_{0}+a_{1} x_{m}+\ldots\right\} d x_{m}=\left\{a_{0}+a_{1} x_{m}+\ldots\right\} \frac{d l}{2 \sqrt{l-l_{m}}},
$$

where $a_{0}, a_{1}, \ldots$ are some 1 -forms with respect to $Q$. Differentiation of (3.5) with respect to $l_{m}$ gives

$$
\frac{\partial B(P, Q)}{\partial l_{m}}=\left\{a_{0}+o(1)\right\} \frac{d l}{4\left(l-l_{m}\right)^{3 / 2}}=\left\{a_{0}+o(1)\right\} \frac{d x_{m}}{2 x_{m}^{2}} .
$$

as $P \rightarrow P_{m}$. Therefore, $\partial B(P, Q) / \partial l_{m}$ is a meromorphic 1 -form with respect to $P$, with the only pole at $P_{m}$ of the second order with leading coefficient

$$
\left.\frac{a_{0}(Q)}{2} \equiv \frac{B(Q, P)}{2 d x_{m}(P)}\right|_{P=P_{m}}
$$

(which is itself a 1 -form with respect to $Q$ ) and vanishing $a$-periods. Taking into account the symmetry of $B(P, Q)$, we get

$$
\frac{\partial B(P, Q)}{\partial l_{m}}=\frac{1}{2}\left\{\left.\frac{B(P, R)}{d x_{m}(R)}\right|_{R=P_{m}}\right\}\left\{\left.\frac{B(Q, R)}{d x_{m}(R)}\right|_{R=P_{m}}\right\}
$$

which is equivalent to (3.4).

Since differentials $B_{m}$ are related to the Bergmann kernel via (3.1), the theorem immediately implies the following useful

Corollary 2 The abelian differential $B_{n}(P)$ and the abelian integral $\emptyset_{n}(P)$ depend on $l_{m}$ (for any $m \neq n$ ) as follows:

$$
\frac{\partial}{\partial l_{m}} B_{n}(P)=\frac{1}{2} b\left(P_{m}, P_{n}\right) B_{m}(P), \quad \frac{\partial}{\partial l_{m}} \varnothing_{n}(P)=\frac{1}{2} b\left(P_{m}, P_{n}\right) \emptyset_{m}(P) .
$$

Now we are in position to formulate the analogs of Euler-Darboux equation in arbitrary genus:

Theorem 6 Consider the following linear system of scalar equations for function $\psi\left(P,\left\{l_{m}\right\}\right)$ :

$$
\frac{d \psi(P)}{d l_{m}}=R_{m} \varnothing_{m}(P)
$$

where $R_{m}$ are some functions of $\left\{l_{m}\right\}$. Denote by $P_{0}$ a point of $E$ such that $l_{0} \equiv \pi\left(P_{0}\right)$ does not depend on $\left\{l_{m}\right\}$. Then the compatibility conditions of the system (3.9) are given by the following equations:

$$
\frac{\partial^{2} f}{\partial l_{m} \partial l_{n}}-\frac{b\left(P_{m}, P_{n}\right)}{2}\left\{\frac{v_{n}}{v_{m}} \frac{\partial f}{\partial l_{m}}+\frac{v_{m}}{v_{n}} \frac{\partial f}{\partial l_{n}}\right\}=0
$$

where $f\left(\left\{l_{m}\right\}\right)=\psi\left(P_{0}\right)$ and

$$
v_{m} \equiv \emptyset_{m}\left(P_{0}\right)=\int_{Q_{0}}^{P_{0}} B_{m} ;
$$

function $b(P, Q)$ is defined by (3.3). 
Proof. Substituting in (3.9) $P=P_{0}$, we see that

$$
R_{m}=\frac{f_{l_{m}}}{\emptyset_{m}\left(P_{0}\right)}
$$

Then the compatibility conditions of the system (3.9) are equivalent to the system of equations

$$
\frac{\partial}{\partial l_{m}}\left\{\frac{\partial f}{\partial l_{n}} \frac{\emptyset_{n}(P)}{\emptyset_{n}\left(P_{0}\right)}\right\}-\frac{\partial}{\partial l_{n}}\left\{\frac{\partial f}{\partial l_{m}} \frac{\emptyset_{m}(P)}{\emptyset_{m}\left(P_{0}\right)}\right\}=0, \quad m \neq n
$$

Using (3.8), we rewrite this equation as follows:

$$
\begin{gathered}
f_{l_{m} l_{n}}\left\{\frac{\emptyset_{n}(P)}{\varnothing_{n}\left(P_{0}\right)}-\frac{\emptyset_{m}(P)}{\emptyset_{m}\left(P_{0}\right)}\right\} \\
+\frac{b\left(P_{m}, P_{n}\right)}{2}\left\{\varnothing_{m}(P) \varnothing_{n}\left(P_{0}\right)-\varnothing_{n}(P) \varnothing_{m}\left(P_{0}\right)\right\}\left\{\frac{f_{l_{n}}}{\varnothing_{n}^{2}\left(P_{0}\right)}+\frac{f_{l_{m}}}{\varnothing_{m}^{2}\left(P_{0}\right)}\right\}=0 .
\end{gathered}
$$

The 1.h.s. of this equation is an abelian integral on $\mathrm{E}$ with respect to argument $P$ with vanishing $a$-periods (since all $a$-periods of differentials $B_{m}(P)$ vanish) and the poles (of the first order) only at $P_{m}$ and $P_{n}$. Since $\varnothing_{m}(P)=-1 / x_{m}+O(1)$ as $P \rightarrow P_{m}$, we immediately see that equations (3.10) are equivalent to absence of poles of (3.12) at $P_{m}$ and $P_{n}$. Therefore, the l.h.s. of (3.12) must be a constant with respect to $P$; choosing $P=P_{0}$ we see that this constant vanishes. We conclude that equations (3.10) indeed provide compatibility of the linear system (3.9).

In analogy to the case of genus zero, now we shall define the tau-function of the system (3.10) and construct its solutions via solutions of the scalar Riemann-Hilbert problem on $\mathrm{E}$.

We define the tau-function of the system (3.10) by the following system of equations:

$$
\frac{\partial}{\partial l_{m}} \ln \tau=\frac{f_{l_{m}}^{2}}{v_{m}^{2}}, \quad \frac{\partial \ln \tau}{\partial \overline{l_{m}}}=0 .
$$

From variational formulas (3.8) and equations (3.10) it follows that

$$
\frac{\partial}{\partial l_{n}}\left\{\frac{f_{l_{m}}^{2}}{v_{m}^{2}}\right\}=b\left(P_{m}, P_{n}\right) \frac{f_{l_{m}} f_{l_{n}}}{v_{m} v_{n}} .
$$

Symmetry of this expression with respect to $m$ and $n$ proves compatibility of equations (3.13).

As well as in the case of genus zero, we can prove the following

Lemma 1 Definition (3.13) of the tau-function can be alternatively rewritten in the following form:

$$
\frac{\partial \ln \tau}{\partial l_{m}}=\left.\frac{1}{2} \operatorname{res}\right|_{P_{m}}\left\{\frac{\left(d_{P} \psi\right)^{2}}{d l}\right\} .
$$

Proof. Choosing the standard local parameter $x_{m}=\sqrt{l-l_{m}}$ near $P_{m}$, we have $d x_{m}=d l / 2 x_{m}$; therefore,

$$
\left.\operatorname{res}\right|_{P_{m}}\left\{\frac{\left(d_{P} \psi\right)^{2}}{d l}\right\}=\frac{1}{2}\left(\frac{\partial \psi}{\partial x_{m}}\right)^{2}\left(P_{m}\right) .
$$


On the other hand, as $P$ belongs to a neighbourhood of $P_{m}$, the linear system (3.9) can be rewritten as follows:

$$
\frac{\partial \psi}{\partial l_{m}}-\frac{1}{2 x_{m}} \frac{\partial \psi}{\partial x_{m}}=\frac{\partial f}{\partial l_{m}} \frac{\emptyset_{m}(P)}{\emptyset_{m}\left(P_{0}\right)}
$$

where we separated the dependence of $\psi$ on $l_{m}$ which comes from $l_{m}$-dependence of $x_{m}$. Taking the residue at $P_{m}$, we get

$$
\frac{\partial \psi}{\partial x_{m}}\left(P_{m}\right)=2 \frac{f_{l_{m}}}{\emptyset_{m}\left(P_{0}\right)}
$$

which implies coincidence of (3.13) and (3.15).

The next theorem provides solutions of the system (3.10).

Theorem 7 Let $l$ be an arbitrary smooth closed contour on $E$ such that its projection on the l-plane $\pi(l)$ is independent of $\left\{l_{m}\right\}$ and $P_{m} \notin l$ for any $m$. Consider on $l$ an arbitrary Hölder-continuous function $h(Q)$ independent of $\left\{l_{m}\right\}$.

Then the function

$$
f=\oint_{l} h(Q) d_{Q} \ln \frac{E\left(P_{0}, Q\right)}{E\left(Q_{0}, Q\right)}
$$

satisfies the system (3.10). Corresponding solution of the linear system (3.9) is given by

$$
\psi(P)=\oint_{l} h(Q) d_{Q} \ln \frac{E(P, Q)}{E\left(Q_{0}, Q\right)} .
$$

Proof. Before proving the statement of the theorem we notice that if the function $h(Q)$ is Höldercontinuous, then $\psi$ (3.17) is a solution of the Riemann-Hilbert problem on the contour $l$ with jump $2 \pi i h(Q)$.

Let us now verify that the functions $f$ and $\psi$ defined by (3.16) and (3.17), satisfy the linear system (3.9):

$$
\psi_{l_{m}}=\frac{\emptyset_{m}(P)}{\emptyset_{m}\left(P_{0}\right)} f_{l_{m}} .
$$

Taking into account representation (3.2) of $\varnothing_{m}(P)$ in terms of prime-forms, in analogy to Rauch formulas given by $\mathrm{Th}$, we see that the Cauchy kernel $d_{Q} \ln \frac{E(P, Q)}{E\left(Q_{0}, Q\right)}$ depends as follows on $l_{m}$ (assuming that all points $P, Q, Q_{0}$ are $l_{m}$-independent):

$$
\frac{\partial}{\partial l_{m}}\left\{d_{Q} \ln \frac{E(P, Q)}{E\left(Q_{0}, Q\right)}\right\}=\frac{1}{2} B_{m}(Q) \emptyset_{m}(P) .
$$

Therefore,

$$
\frac{\psi_{l_{m}}(P)}{\emptyset_{m}(P)}=\frac{1}{2} \oint_{l} h(Q) B_{m}(Q)
$$

Independence of this expression of $P$ proves (3.18). Therefore, the function $f$ satisfies the compatibility conditions of (3.18) i.e. the equations (3.10).

We notice that, using superposition principle for the systems (3.10), we can substitute integration over contour in (3.16) by integration with respect to an arbitrary $\left\{l_{m}\right\}$-independent measure with compact support; the only condition we need to impose on this measure is commutativity of integration in (3.16) with differentiation with respect to $\left\{l_{m}\right\}$. 


\subsection{Systems of higher rank}

Here we consider coverings of genus $g \geq 2$. To define a natural rank $r$ analogs of equations (3.10), it is natural to start from generalization of the zero curvature representation (2.11) to higher genus:

$$
\frac{\partial \Psi}{\partial l_{m}}=U_{m} \Psi
$$

where the $r \times r$ "Lax matrix" $U_{m}\left(P,\left\{l_{n}\right\}\right), P \in \mathrm{E}$ has only one singularity on $\mathrm{E}$, which is a simple pole at the ramification point $P_{m}$. We shall assume that $\operatorname{tr} U_{m}=0$ (this condition can be always satisfied by normalization of function $\left.\Psi: \Psi \rightarrow[\operatorname{det} \Psi]^{-1 / r} \Psi\right)$. As well as in $\operatorname{rank} 1$, the "Lax matrix" $U_{m}(P)$ can not be single-valued on $\mathrm{E}$. In rank 1 matrix $U_{m}$ has additive twists along basic cycles on $\mathrm{E}$, but $d_{P} U_{m}(P)$ is a single-valued meromorphic differential on $\mathrm{E}$ with pole of second order at $P_{m}$.

In higher rank this is not enough - it is necessary to introduce more degrees of freedom, and allow $d_{P} U_{m}$ to suffer similarity transformation under tracing along each topologically non-trivial closed contour on $\mathrm{E}$ (this transformation may be $\left\{l_{m}\right\}$-dependent itself).

More precisely, consider a stable vector bundle $\chi$ of rank $r$ and degree $d$ over Ł. Require $d_{P} U_{m}$ to be a meromorphic section of the bundle $a d_{\chi} \otimes K$ with quadratic pole at the ramification point $P_{m}(K$ is the canonical line bundle), i.e.

$$
d_{P} U_{m} \in H^{0}\left(\mathrm{E}, a d_{\chi} \otimes K\left(2 P_{m}\right)\right)
$$

according to terminology proposed by Hitchin [1], $d_{P} U_{m}$ is called the meromorphic Higgs field.

According to Narasimhan-Seshadri theorem [23, the stable bundle $\chi$ is characterized by the set of unitary constant (independent of a point $P \in \mathrm{E}$ ) matrices $\chi_{a_{1}}, \ldots, \chi_{a_{g}}, \chi_{b_{1}}, \ldots, \chi_{b_{g}}$, satisfying one relation

$$
\prod_{\alpha=1}^{g} \chi_{a_{j}} \chi_{b_{j}} \chi_{a_{j}}^{-1} \chi_{b_{j}}^{-1}=\exp \left\{2 \pi i \frac{d}{r}\right\} .
$$

Stability of the bundle $\chi$ implies $h^{0}\left(a d_{\chi}\right)=0$ i.e. the bundle $a d_{\chi}$ does not have any holomorphic section. On the other hand, $h^{0}\left(a d_{\chi} \otimes K\right)=(g-1)\left(r^{2}-1\right)$; therefore, fixing the singular part of $d_{P} U_{m}$ at the ramification point $P_{m}$ :

$$
d_{P} U_{m}(P)=\left(-\frac{R_{m}}{x_{m}^{2}}+O(1)\right) d x_{m} \quad \text { as } \quad P \rightarrow P_{m}
$$

we define $d_{P} U_{m}$ up to an arbitrary linear combination of $(g-1)\left(r^{2}-1\right)$ holomorphic sections of $a d_{\chi} \otimes K$. In rank 1 case we fixed $d_{P} U_{m}$ uniquely imposing the normalization condition of vanishing of all $a$-periods of $d_{P} U_{m}$; unfortunately, such simple normalization in higher rank is not known to the authors.

The "Lax matrix" $U_{m}$ is uniquely defined by $d_{P} U_{m}$ inside of the fundamental polygon $\hat{\mathrm{E}}$ of $\mathrm{E}$ if we fix a normalization point $P_{0} \in \hat{\mathrm{E}}$ and assume that $U_{m}\left(P_{0}\right)=0$. The only singularity of the matrix $U_{m}$ is the simple pole at $P_{m}$ of the form

$$
U_{m}(P)=\frac{R_{m}}{x_{m}}+O(1) \quad \text { as } \quad P \rightarrow P_{m}
$$

generically $U_{m}$ has both multiplicative and additive non-single-valuedness along any cycle $\gamma \in \pi_{1}(\mathrm{E})$ :

$$
U_{m}\left(P^{\gamma}\right)=\chi_{\gamma} U_{m}(P) \chi_{\gamma}^{-1}+C_{m}^{\gamma}
$$


since $d_{P} U_{m}\left(P^{\gamma}\right)=\chi_{\gamma} d_{P} U_{m}(P) \chi_{\gamma}^{-1}$, the "additive twists" $C_{m}^{\gamma}$ don't depend on $P$.

The compatibility conditions of the linear system (3.20) are given by:

$$
F_{m n}(P)=0
$$

where $F_{m n}(P)$ is the curvature:

$$
F_{m n}(P)=\frac{\partial U_{m}}{\partial l_{n}}-\frac{\partial U_{n}}{\partial l_{m}}+\left[U_{m}, U_{n}\right]
$$

To rewrite the compatibility conditions (3.23) in terms of variables depending only on $\left\{l_{m}\right\}$ (and not on the point $P$ of the covering $\mathrm{E})$, we consider the coefficients of the Taylor series of $U_{m}(P)$ at the ramification point $P_{n}, n \neq m$ :

$$
U_{m}(P)=S_{m n}+T_{m n} x_{n}+O\left(x_{n}^{2}\right) .
$$

Then non-singularity of $F_{m n}$ at $P_{m}$ is equivalent to the condition

$$
\frac{\partial R_{m}}{\partial l_{n}}+\frac{1}{2} T_{n m}+\left[R_{m}, S_{n m}\right]=0, \quad m \neq n .
$$

The non-singularity of $F_{m n}$ is insufficient for its vanishing, since $F_{m n}(P)$ has both multiplicative and additive twists along topologically non-trivial loops. However, if we require that the "additive twists" $C_{m}^{\gamma}$ from (3.22) are related to matrices $\chi_{\gamma}$ via equations

$$
\frac{\partial \chi_{\gamma}}{\partial l_{m}} \chi_{\gamma}^{-1}=C_{m}^{\gamma}
$$

we observe that transformation (3.22) of the Lax matrices $U_{m}$ is nothing but the gauge transformation of connection 1-form $\sum_{m=1}^{M} U_{m} d l_{m}$ by the matrix $\chi_{\gamma}\left(\left\{l_{m}\right\}\right)$. Then the curvature coefficient $F_{m n}$ transforms as follows:

$$
F_{m n}\left(P^{\gamma}\right)=\chi_{\gamma} F_{m n}(P) \chi_{\gamma}^{-1}
$$

i.e. $F_{m n} \in H^{0}\left(a d_{\chi}\right)$; thus $F_{m n}=0$.

We conclude that the compatibility conditions of the linear system (3.20) in arbitrary rank $r$ and any genus $g \geq 2$ are given by the system of equations (3.25) and (3.26). The new feature in comparison with the genus zero case is that we get another degree of freedom - the stable bundle $\chi$ must itself depend on the branch points $l_{m}$ according to equations (3.26) (since generic vector bundle over $\mathrm{E}$ is stable, generically evolution (3.26) preserves stability of $\chi$ at least locally, in a neighbourhood of a given stable bundle).

Obviously, without any normalization of the twisted 1-form $d_{P} U_{m}$ the coefficients $S_{m n}, T_{m n}$ and additive twists $C_{m}^{\gamma}$ are not uniquely determined by the set of residues $R_{m}$ and matrices $\chi_{\gamma}$. Therefore, the number of equations (3.25),(3.26) is substantially smaller than the number of variables.

A possible way to define $d_{P} U_{m}$ uniquely is to make use of one of meromorphic bidifferentials $W(P, Q)$ on $\mathrm{E} \times \mathrm{\textrm {E }}$ whose existence is provided by the following lemma:

Lemma 2 There exists meromorphic bidifferential $W(P, Q)$ on $E \times E$ satisfying the following conditions: 
1. On the diagonal $P=Q$ the bidifferential $W(P, Q)$ has second order pole with biresidue equal to $r^{2} \times r^{2}$ matrix $\Pi$ (which is the permutation matrix in $\mathbb{C}^{r} \otimes \mathbb{C}^{r}$ ):

$$
W(P, Q)=\left\{\frac{\Pi}{\left(x_{P}-x_{Q}\right)^{2}}+O(1)\right\} d x_{P} d x_{Q} .
$$

2. Symmetry condition:

$$
W(P, Q)=\Pi W(Q, P) \Pi .
$$

3. Automorphy conditions: for any $\gamma \in \pi_{1}(E)$ we have

$$
\begin{aligned}
& W\left(P^{\gamma}, Q\right)=\stackrel{1}{\chi}_{\gamma} W(P, Q) \stackrel{1_{\chi}^{-1}}{\gamma}, \\
& W\left(P, Q^{\gamma}\right)=\stackrel{2}{\chi}_{\gamma} W(P, Q) \stackrel{2}{\chi}_{\gamma}^{-1},
\end{aligned}
$$

where for any linear operator $A$ in $\mathbb{C}^{r}$ we denote by $\stackrel{1}{A}$ and $\stackrel{2}{A}$ the operators $A \otimes I$ and $I \otimes A$ in $\mathbb{C}^{r} \otimes \mathbb{C}^{r}$, respectively.

The relation (3.30) is obviously a corollary of (3.29) and the symmetry requirement (3.28). Equivalently, relations (3.29), (3.30) mean that $W(P, Q)$ belongs to $H^{0}\left(a d_{\chi} \otimes K(2 Q)\right)$ with respect to its first argument, and to $H^{0}\left(a d_{\chi} \otimes K(2 P)\right)$ with respect to its second argument.

Proof. Existence of bidifferential $W_{0}(P, Q)$ satisfying only (3.27) and automorphy properties (3.29), (3.30), can be proved similarly to, say, existence of Schiffer kernel corresponding to the bundle $a d_{\chi}$ (see for example [8]). To construct bidifferential $W(P, Q)$ which satisfies in addition the symmetry condition (3.28), suppose that $F(P, Q) \equiv W_{0}(P, Q)-\Pi W_{0}(Q, P) \Pi$ does not vanish; this is a holomorphic section of $a d_{\chi} \otimes K$ with respect to $P$ and holomorphic section of $a d_{\chi} \otimes K$ with respect to $Q$. Obviously,

$$
\Pi F(P, Q) \Pi=-F(Q, P) .
$$

Now define $W(P, Q)=W_{0}(P, Q)-\frac{1}{2} F(P, Q)$. Simple calculation shows that it satisfies (3.28); other required properties are inherited from $W_{0}(P, Q)$.

We proved existence of $W(P, Q)$; obviously this bidifferential is not unique: we can add to $W(P, Q)$ an arbitrary linear combination of bilinear products of holomorphic sections $f_{k}(P)$ of $a d_{\chi} \otimes K$, satisfying the symmetry condition (3.28); this is a linear combination of the form

$$
\sum_{j, k=1}^{(g-1)\left(r^{2}-1\right)} \alpha_{j k}\left\{\stackrel{1}{f}_{k}(P) \stackrel{2}{f_{j}}(Q)+\stackrel{1}{f_{j}}(P) \stackrel{2}{f_{k}}(Q)\right\}
$$

with arbitrary $\alpha_{j k} \in \mathbb{C}$.

Let us fix the bidifferential $W(P, Q)$ in some way (for example, according to remark 3 below). Then the Higgs fields $d_{P} U_{m}$ can be defined as follows:

$$
d_{P} U_{m}(P)=\left.\frac{\operatorname{tr}\{\stackrel{12}{W}(P, Q) \stackrel{2}{R} m\}}{d x_{m}(Q)}\right|_{Q=P_{m}} .
$$


Now all variables $S_{m n}, T_{m n}, C_{m}^{\gamma}$ become functionals of the residues $R_{m}$, branch points $l_{m}$ and matrices $\chi_{\gamma}$; the number of variables $\left(\chi_{\gamma}, R_{m}\right)$ in the system (3.25), (3.26) coincides with the number of equations.

The natural definition of tau-function, in agreement with (2.19), looks as follows:

$$
\frac{\partial}{\partial l_{m}} \ln \tau=\left.\frac{1}{2} \operatorname{res}\right|_{P_{m}} \frac{\operatorname{tr}\left(d_{P} \Psi \Psi^{-1}\right)^{2}}{d l} .
$$

Consistency of the definition (3.32) is provided by the following

Lemma 3 Let function $\Psi$ solve the linear system (3.20) with Lax matrices $U_{m}$ satisfying condition $U_{m}\left(P_{0}\right)=0$ for some $P_{0} \in E$, and conditions 3.31). Then the 1-form

$$
\sum_{m=1}^{M}\left\{\left.\operatorname{res}\right|_{P_{m}} \frac{\operatorname{tr}\left(d_{P} \Psi \Psi^{-1}\right)^{2}}{d l}\right\} d l_{m}
$$

is closed.

Proof. Consider the Taylor series of $\Psi(P)$ in a neighbourhood of $P_{m}$ :

$$
\Psi(P)=\Psi_{0}+x_{m} \Psi_{1}+O\left(x_{m}^{2}\right) ;
$$

then

$$
\Psi_{x_{m}} \Psi^{-1}=\Psi_{1} \Psi_{0}^{-1}+O(1)
$$

and

$$
\Psi_{l_{m}} \Psi^{-1}=-\frac{1}{2 x_{m}} \Psi_{1} \Psi_{0}^{-1}+O(1)
$$

Therefore,

$$
R_{m}=-\frac{1}{2} \Psi_{1} \Psi_{0}^{-1}
$$

and

$$
\left.\frac{1}{2} \mathrm{res}\right|_{P_{m}} \frac{\operatorname{tr}\left(d_{P} \Psi \Psi^{-1}\right)^{2}}{d l}=\left.\frac{1}{2} \operatorname{res}\right|_{P_{m}} \frac{\operatorname{tr}\left(-2 R_{m} d x_{m}\right)^{2}}{2 x_{m} d x_{m}}=\operatorname{tr} R_{m}^{2}
$$

Now we have to make sure that the derivative $\frac{\partial}{\partial l_{n}} \operatorname{tr} R_{m}^{2}$ is symmetric under the interchanging of $m$ and $n$ :

$$
\frac{\partial}{\partial l_{n}} \operatorname{tr} R_{m}^{2}=-2 \operatorname{tr} R_{m}\left\{\frac{1}{2} T_{n m}+\left[R_{m}, S_{n m}\right]\right\}=-\operatorname{tr} R_{m} T_{n m}
$$

As $P \rightarrow P_{m}$, we have:

$$
\operatorname{tr}\left\{d_{P} U_{m}(P) d_{P} U_{n}(P)\right\}=\left(-\operatorname{tr} R_{m} T_{n m}+\ldots\right)\left(\frac{d x_{m}}{x_{m}}\right)^{2} ;
$$

symmetry of (3.34) is thus equivalent to relation

$$
\text { bires }\left.\right|_{P_{m}} \operatorname{tr}\left\{d_{P} U_{m} d_{P} U_{n}\right\}=\text { bires }\left.\right|_{P_{n}} \operatorname{tr}\left\{d_{P} U_{m} d_{P} U_{n}\right\} .
$$

Let us rewrite the l.h.s. of this relation in terms of bidifferential $W(P, Q) \equiv w(P, Q) d x_{P} d x_{Q}$ according to (3.31), taking into account the behaviour (3.27) of $W(P, Q)$ on the diagonal $P=Q$ :

$$
\text { bires }\left.\right|_{P_{m}} \operatorname{tr}\left\{d_{P} U_{m} d_{P} U_{n}\right\}=\left.\operatorname{bires}\right|_{P_{m}} \operatorname{tr}\left\{\stackrel{3}{\operatorname{tr}}\left\{\stackrel{3}{R} \underset{m}{w} \underset{13}{w}\left(P, P_{m}\right)\right\} \stackrel{2}{\operatorname{tr}}\left\{\stackrel{2}{R_{n}} \stackrel{12}{w}\left(P, P_{n}\right)\right\}\right\}\left(d x_{m}(P)\right)^{2}
$$




$$
=\stackrel{1}{2} \underset{\operatorname{tr}}{2}\left\{\stackrel{1}{R_{m}} \stackrel{2}{R} \underset{n}{w} \underset{w}{(2}\left(P_{m}, P_{n}\right)\right\}
$$

Similarly,

$$
\begin{gathered}
\text { bires } \left.\left.\right|_{P_{m}} \operatorname{tr}\left\{d_{P} U_{m} d_{P} U_{n}\right\}=\operatorname{tr} \underset{\operatorname{tr}}{2} \underset{R_{m}}{2} \stackrel{1}{R}_{n} \stackrel{12}{w}\left(P_{n}, P_{m}\right)\right\} \\
=\stackrel{1}{\operatorname{tr} \operatorname{tr}}\left\{\stackrel{2}{R_{m}} \stackrel{1}{R_{n}} \Pi \stackrel{12}{w}\left(P_{m}, P_{n}\right) \Pi\right\}=\underset{\operatorname{tr}}{\operatorname{tr}}\left\{\stackrel{1}{R_{m}} \stackrel{2}{R_{n}} \underset{12}{w}\left(P_{m}, P_{n}\right)\right\},
\end{gathered}
$$

coinciding with (3.35); the last equality in (3.36) follows from the symmetry property (3.28) of $W(P, Q)$.

Remark 2 More explicit treatment is, as usual, possible for elliptic coverings $g=1$, when the bundle $a d_{\chi}$ can possess meromorphic sections with single simple pole. In this case the additive twists $C_{m}^{\gamma}$ are absent, and monodromy matrices $\chi_{\gamma}$ of the bundle $\chi$ can be chosen to be independent of $\left\{l_{m}\right\}$. In this case the above construction can be nicely rewritten in terms of elliptic r-matrix [27].

Remark 3 In rank 1 case we have fixed the bidifferential $W(P, Q)$ by the requirement that all of its $a$-periods vanish; then $W(P, Q)$ coincides with Bergmann kernel. This kind of normalization is not possible in higher rank due to non-invariance of $W(P, Q)$ under tracing along topologically non-trivial loops on $\mathrm{€}$. However, in rank 1 case there exists another way to fix $W(P, Q)$ uniquely: one can require that v.p. $\iint_{\mathrm{E}} W(P, Q) \overline{w_{\alpha}(Q)}=0$ for any holomorphic differential $w_{\alpha}$ (in this case $W(P, Q)$ is called the Schiffer kernel). These conditions have natural higher rank analog [8]. Namely, we can require that v.p. $\iint_{€} \stackrel{2}{\operatorname{tr}}\left\{\underset{W}{W}(P, Q) f_{k}^{\dagger}(Q)\right\}=0$ for any $f_{k} \in H^{0}\left(a d_{\chi} \otimes K\right)$; due to unitarity of the matrices $\chi_{\alpha}$ the integrand is a $(1,1)$-form on $\mathrm{E}$. If the biresidue at $P=Q$ is chosen to be the unit matrix $I \otimes I$ instead of permutation matrix $\Pi$, our definition of $W(P, Q)$ would give the Schiffer kernel corresponding to the bundle $a d_{\chi}\left[\underline{8}\right.$. This normalization of $W(P, Q)$ leads to the following normalization of $d_{P} U_{m}$ : v.p. $\iint_{€} \operatorname{tr}\left\{d_{P} U_{m} f_{k}^{\dagger}\right\}=0$ for any $f_{k} \in H^{0}\left(a d_{\chi} \otimes K\right)$.

We notice that, in contrast to Bergmann kernel in rank 1, this normalization of $W(P, Q)$ makes it non-holomorphic function of "moduli" $l_{m}$; therefore, in principle, the complete system of equations should contain also equations with respect to $\left\{l_{m}^{-}\right\}$We shall discuss these aspects in more details in further publication.

Relationship to isomonodromy deformations in higher genus. The link between the genus zero systems (2.13) and isomonodromic deformations on Riemann sphere discussed in Sect 2.5 can be extended to arbitrary genus (we outline it here for $g \geq 2$ ). Let us briefly describe the isomonodromic deformations on the covering $€$. Consider a stable bundle $\chi$ characterized by a set of unitary matrices $\chi_{\gamma}$ for any $\gamma \in \pi_{1}(\mathrm{E})$; consider also a divisor $Q=Q_{1}+\cdots+Q_{L}$ on $\mathrm{E}$.

Introduce a Higgs field $A(P)$ which is allowed to have simple poles at the points $Q_{1}, \ldots, Q_{L}$, i.e. $A \in H^{0}\left(a d_{\chi} \otimes K(Q)\right)$; suppose that $\operatorname{tr} A(P)=0$. The higher genus analog of the linear differential equation (2.35) looks as follows:

$$
d_{P} \Psi=A(P) \Psi
$$

where function $\Psi$ has unit determinant and satisfies the initial condition $\Psi\left(P_{0}\right)=I$ at some point $P_{0} \in \mathrm{E}$. 
In analog to genus 0 case, function $\Psi$ has regular singularities at the points $Q_{1}, \ldots, Q_{L}$ with some monodromy matrices $M_{k}$, i.e. under tracing around $Q_{k}$ the function $\Psi(P)$ transforms as follows:

$$
\Psi(P) \rightarrow \Psi(P) M_{k}
$$

Under tracing along basic cycles of $€$ the function $\Psi$ gains left multipliers given by the matrices $\chi^{\gamma}$; in addition, it may gain the right multipliers $M_{a_{\alpha}}, M_{b_{\alpha}}$, which are analogs of monodromy matrices $M_{k}$ :

$$
\Psi\left(P^{a_{\alpha}}\right)=\chi_{a_{\alpha}} \Psi(P) M_{a_{\alpha}}, \quad \Psi\left(P^{b_{\alpha}}\right)=\chi_{b_{\alpha}} \Psi(P) M_{b_{\alpha}} .
$$

The monodromy matrices $M_{k}, M_{a_{\alpha}}, M_{b_{\alpha}}$ generate a $S L(r)$ "monodromy" representation of the fundamental group $\pi_{1}\left(\mathrm{E} \backslash\left\{Q_{k}\right\}\right)$.

If we now assume that all the monodromy matrices $M_{k}, M_{a_{\alpha}}, M_{b_{\alpha}}$ are independent of the branch points $l_{m}$ and projections $\mu_{k}=\pi\left(Q_{k}\right)$ of the regular singularities, then function $\Psi$ satisfies the deformation equations

$$
\begin{gathered}
\Psi_{\mu_{k}}=V_{k} \Psi, \\
\Psi_{l_{m}}=U_{m} \Psi,
\end{gathered}
$$

where matrix $V_{k}$ has simple pole at the regular singularity $Q_{k}$; matrix $U_{m}$ has simple pole at the ramification point $P_{m}$; these matrices transform as follows under the tracing along any $\gamma \in \pi_{1}(\mathrm{E})$ :

$$
\begin{gathered}
V_{k}\left(P^{\gamma}\right)=\chi_{\gamma} V_{k}(P) \chi_{\gamma}^{-1}+\left(\chi_{\gamma}\right)_{\mu_{k}} \chi_{\gamma}^{-1}, \\
U_{m}\left(P^{\gamma}\right)=\chi_{\gamma} U_{m}(P) \chi_{\gamma}^{-1}+\left(\chi_{\gamma}\right)_{l_{m}} \chi_{\gamma}^{-1} .
\end{gathered}
$$

Obviously, the part (3.39) of the deformation equations is nothing but the linear system (3.20) introduced above; therefore, the isomonodromic deformations in higher genus correspond, as well as in genus zero, to a subset of solutions of the integrable systems (3.25), (3.26).

\section{Systems of rank 1, Darboux-Egoroff metrics and systems of hy- drodynamic type}

\subsection{Darboux-Egoroff metrics}

It turns out that each solution of the rank 1 system (3.10) defines a flat diagonal (pseudo-)metric in $\mathbb{C}^{M}$ (which gives rise to a flat diagonal Darboux-Egoroff metric in $\mathbb{R}^{M}$ if additional reality and positivity conditions are imposed). In the sequel we shall (in agreement with previous works on the subject) use the term "Darboux-Egoroff metric" for such pseudo-metrics in $\mathbb{C}^{M}$.

For diagonal (pseudo-) metric

$$
d s^{2}=\sum_{m=1}^{M} g_{m m} d l_{m}^{2}
$$

the Christoffel symbols are given by

$$
\Gamma_{m n}^{k}=0, \quad \Gamma_{n m}^{n}=\partial_{l_{m}} \ln \sqrt{g_{n n}}, \quad m \neq n \neq k
$$


they are related as follows to rotation coefficients $\beta_{m n}$ :

$$
\beta_{m n}=\frac{\sqrt{g_{m m}}}{\sqrt{g_{n n}}} \Gamma_{m n}^{m} \equiv \frac{\partial_{l_{n}} \sqrt{g_{m m}}}{\sqrt{g_{n n}}}, \quad m \neq n
$$

The metric (4.1) is flat iff the rotation coefficients satisfy the following equations:

$$
\frac{\partial \beta_{m n}}{\partial l_{l}}=\beta_{m l} \beta_{l n}
$$

for any distinct $l, m, n$, and each of $\beta_{m n}$ is invariant with respect to simultaneous shifts along all $\left\{l_{k}\right\}$ :

$$
\sum_{k=1}^{M} \frac{\partial \beta_{m n}}{\partial l_{k}}=0
$$

If in addition the rotation coefficients are symmetric, $\beta_{m n}=\beta_{n m}$, which is equivalent to relation $\partial_{m} g_{n n}=\partial_{n} g_{m m}$, then there exists potential $U$ such that

$$
g_{m m}=\frac{\partial U}{\partial l_{m}}
$$

and the metric (4.1) is called the Darboux-Egoroff metric.

We shall now prove that each solution of the system (3.10) corresponds to Darboux-Egoroff metric.

Theorem 8 Let $f$ be an arbitrary solution of the system (3.9), and $\tau\left(\left\{l_{m}\right\}\right)$ be the corresponding tau-function defined by (3.13) or (3.15). Then metric (4.1) with

$$
g_{m m}=\frac{\partial \ln \tau}{\partial l_{m}}
$$

is a Darboux-Egoroff metric in $\mathbb{C}^{M}$.

Proof. Let us compute the rotation coefficients of the metric 4.1). From the definition of tau-function (3.13) we have

$$
\sqrt{\frac{\partial \ln \tau}{\partial l_{m}}}=\frac{f_{l_{m}}}{v_{m}}
$$

using the expression (3.14) for the second derivative of the tau-function, we find

$$
\beta_{m n}=\frac{1}{2} \frac{(\ln \tau)_{l_{m} l_{n}}}{\sqrt{(\ln \tau)_{l_{m}}} \sqrt{(\ln \tau)_{l_{n}}}}=\frac{1}{2} b\left(P_{m}, P_{n}\right) .
$$

These functions satisfy the equations (4.4) as a corollary of variational formulas (3.4).

It remains to prove that each $b\left(P_{m}, P_{n}\right)$ satisfies equations (4.5). We shall use invariance of the Bergmann kernel $B(P, Q)$ with respect to biholomorphic maps. Let us consider the branched covering $\mathrm{E}^{\epsilon}$ which is obtained by small $\epsilon$-shift of all the ramification points $P_{m}$ in $l$-plane i.e. the projections of branch points $P_{m}^{\epsilon}$ of $\mathrm{E}^{\epsilon}$ on $l$-plane are equal to $l_{m}^{\epsilon}=l_{m}+\epsilon ; B^{\epsilon}$ is the Bergmann kernel on $\mathrm{E}^{\epsilon}$. Denote the projections of points $P$ and $Q$ on $l$-plane by $l$ and $\mu$, respectively. Define the point $P^{\epsilon}$ to be the point lying on the same sheet as $P$ and having projection $l+\epsilon$ on $l$-plane; in the same way point $Q^{\epsilon}$ 
belongs to the same sheet as $Q$ and has projection $\mu+\epsilon$ on $l$-plane. Since $\mathrm{L}^{\epsilon}$ can be holomorphically mapped to $\mathrm{E}$ by transformation $l \rightarrow l+\epsilon$ on all the sheets, we have

$$
B^{\epsilon}\left(P^{\epsilon}, Q^{\epsilon}\right)=B(P, Q)
$$

Assuming that $P$ belongs to a neighbourhood of the branch point $P_{m}$, and $Q$ belongs to a neighbourhood of the branch point $P_{n}$, we can write down the respective local parameters as $x_{m}(P)=\sqrt{l-l_{m}}$ and $x_{n}(Q)=\sqrt{\mu-l_{n}}$. These parameters are obviously invariant with respect to simultaneous $\epsilon$-shifts of all $\left\{l_{m}\right\}, l$ and $\mu$ : $x_{m}^{\epsilon}\left(P^{\epsilon}\right)=x_{m}(P)$ and $x_{n}^{\epsilon}\left(Q^{\epsilon}\right)=x_{n}(Q)$. Therefore, equality (4.8) induces the same relation between $b(P, Q) \equiv B(P, Q) / d x(P) d x(Q)$ :

$$
b^{\epsilon}\left(P^{\epsilon}, Q^{\epsilon}\right)=b(P, Q) .
$$

Assuming now that $P=P_{m}$ and $Q=P_{n}$ and differentiating (4.9) with respect to $\epsilon$ at $\epsilon=0$, we come to (4.5).

Of course, besides simultaneous translations, the Bergmann kernel is invariant with respect to any other Möbius transformation of $l$-plane performed simultaneously on all the sheets of $€$. We can use this invariance to obtain two relations, corresponding to other one-parametric families of Möbius transformations.

Proposition 1 The rotation coefficients (4.7) of the Darboux-Egoroff metric (4.1) satisfy the following relations:

$$
\begin{gathered}
\left\{\sum_{k=1}^{M} l_{k} \frac{\partial}{\partial l_{k}}\right\} \beta_{m n}=-\beta_{m n}, \\
\left\{\sum_{k=1}^{M} l_{k}^{2} \frac{\partial}{\partial l_{k}}\right\} \beta_{m n}=-\left(l_{n}+l_{m}\right) \beta_{m n},
\end{gathered}
$$

Proof. Relation (4.10) corresponds to invariance of the Bergmann kernel under simultaneous dilatation on every sheet of $€$ i.e. to the transformations

$$
l_{m} \rightarrow(1+\epsilon) l_{m}, \quad l \rightarrow(1+\epsilon) l, \quad \mu \rightarrow(1+\epsilon) \mu .
$$

The new feature in comparison with the proof of relation (4.5) is that the local parameters are now dependent on $\epsilon$ :

$$
x_{m}^{\epsilon}\left(P^{\epsilon}\right) \equiv\left[(1+\epsilon) l-(1+\epsilon) l_{m}\right]^{1 / 2}=(1+\epsilon)^{1 / 2} x_{m}(P)
$$

and

$$
x_{n}^{\epsilon}\left(Q^{\epsilon}\right) \equiv\left[(1+\epsilon) \mu-(1+\epsilon) l_{n}\right]^{1 / 2}=(1+\epsilon)^{1 / 2} x_{n}(Q) ;
$$

therefore, invariance $B^{\epsilon}\left(P^{\epsilon}, Q^{\epsilon}\right)=B(P, Q)$ of the Bergmann kernel translates on the level of $b(P, Q)$ as follows:

$$
(1+\epsilon) b^{\epsilon}\left(P^{\epsilon}, Q^{\epsilon}\right)=b(P, Q) .
$$

Differentiating this relation with respect to $\epsilon$ at $\epsilon=0$ via the chain rule and choosing $P=P_{m}$ and $Q=P_{n}$, we get (4.10). 
In a similar way we can deduce (4.11) from invariance of the Bergmann kernel with respect to the one-parametric family of transformations

$$
l \rightarrow l^{\epsilon}=\frac{l}{1+\epsilon l}
$$

on each sheet of .

Relation (4.10) for the rotation coefficients can be found in [6]; relation (4.11) seems to be new. We also notice that all primary differentials used in [6] to construct Frobenius manifolds from Hurwitz spaces can be obtained from solutions (3.16) of the systems (3.10) by appropriate specification of the contour $l$ and the function $h(Q)$.

\subsection{Systems of hydrodynamic type}

According to well-known results (see, for example, review [26]), to each Darboux-Egoroff metric one can associate a class of diagonal systems of hydrodynamic type

$$
\frac{\partial l_{m}}{\partial x}=V_{m} \frac{\partial l_{m}}{\partial t}
$$

where functions $V_{m}\left(\left\{l_{k}\right\}\right)$ (the "characteristic speeds") are related to Christoffel symbols (4.2) of the metric (4.1) via system of differential equations:

$$
\partial_{m} V_{n}=\Gamma_{n m}^{n}\left(V_{m}-V_{n}\right)
$$

Compatibility of equations (4.14) is provided by equations (4.4) for rotation coefficients.

Let us choose some solution of the system (3.10) parametrized by an arbitrary function $h(P)$ on contour $l$ (3.16). Then the metric coefficients (4.6) are given by:

$$
g_{m m}=\left\{\frac{1}{2} \oint_{l} h(Q) B_{m}(Q)\right\}^{2} \text {. }
$$

The Christoffel symbols of this metric look as follows:

$$
\Gamma_{n m}^{n}=\beta_{m n} \frac{\oint_{l} h(P) B_{m}(P)}{\oint_{l} h(P) B_{n}(P)} .
$$

Solutions of equations (4.14) for these Christoffel symbols are described by the following proposition $^{2}$.

Proposition 2 Let $h_{1}(P)$ be an arbitrary Hölder-continuous and independent of $\left\{l_{m}\right\}$ function on contour $l$. Then the functions

$$
V_{m}=\frac{\oint_{l} h_{1}(P) B_{m}(P)}{\oint_{l} h(P) B_{m}(P)}
$$

satisfy system (4.14) with Christoffel coefficients given by (4.2).

\footnotetext{
${ }^{2}$ Formula 4.17) is due to T.Grava (private communication); similar formula for the case of Whitham equations was obtained in [18].
} 
The proof of this proposition is a simple calculation based on Rauch variational formulas for differentials $B_{m}$ :

$$
\frac{\partial}{\partial l_{n}} B_{m}(P)=\beta_{m n} B_{n}(P)
$$

To construct solutions of the system (4.13) with characteristic speeds (4.17) one needs to use the following theorem by Tsarev [26]:

Theorem 9 Let functions $V_{m}\left(l_{1}, \ldots, l_{M}\right)$ satisfy equations 4.14). Then system of equations

$$
\Phi_{m}\left(\left\{l_{k}\right\}\right)=t+V_{m}\left(\left\{l_{k}\right\}\right) x
$$

defines implicit solution $\left\{l_{m}(x, t)\right\}$ of the system of hydrodynamic type 4.13), where $\Phi_{m}\left(l_{1}, \ldots, l_{M}\right)$ is an arbitrary solution of the system of differential equations

$$
\frac{\partial_{n} \Phi_{m}}{\Phi_{m}-\Phi_{n}}=\frac{\partial_{n} V_{m}}{V_{m}-V_{n}}
$$

for $m, n=1, \ldots, M$.

To apply the hodograph method to any of these systems we need to solve also the system of equations (4.20) for functions $\Phi_{m}$. Obviously, the solution is given by same formulas as solution of equations (4.14) for functions $V_{m}$, but with another arbitrary Hölder-continuous function $h_{2}(P)$ independent of $\left\{l_{m}\right\}$ :

$$
\Phi_{m}=\frac{\oint_{l} h_{2}(P) B_{m}(P)}{\oint_{l} h(P) B_{m}(P)} .
$$

For each choice of $h_{2}(P)$ the system of equations (4.19) defines the implicit solution $\left\{l_{m}(x, t)\right\}$ of the system of hydrodynamic type (4.13).

We proved the following

Theorem 10 Consider system of hydrodynamic type (4.13), where velocities $V_{m}$ are given by formula (4.17) with arbitrary Hölder-continuous and independent of $\left\{l_{m}\right\}$ functions $h(P)$ and $h_{1}(P)$ on contour $l$. Let $h_{2}(P)$ be another arbitrary and independent of $\left\{l_{m}\right\}$ Hölder-continuous function on contour $l$. Then system of $M$ equations for $M$ variables $\left\{l_{m}(x, t)\right\}_{m=1}^{M}$

$$
\oint_{l}\left\{h_{2}(P)+h(P) t+h_{1}(P) x\right\} B_{m}(P)=0, \quad m=1, \ldots, M
$$

defines implicit solution $\left\{l_{m}(x, t)\right\}$ of the system 4.13).

As before, the condition of Hölder-continuity of functions $h, h_{1}$ and $h_{2}$ on contour $l$ can be relaxed. Namely, we can substitute the contour $l$ by an arbitrary subset of $\mathrm{E}$, and define on this subset three arbitrary measures independent of $\left\{l_{m}\right\}$; the only requirement one needs to impose is commutativity of integration in (4.22) with differentiation with respect to $\left\{l_{m}\right\}$. 


\section{Summary and outlook}

In this paper we propose a new class of integrable systems of partial differential equations associated to spaces of generic rational maps of fixed degree. For maps of degree two such systems give rise to the Ernst equation from general relativity; for maps of higher degree our systems realize the scheme of deformation of autonomous integrable systems proposed by Burtsev, Mikhailov and Zakharov. We introduce the notion of the tau-function of the new systems, and describe their relationship to the matrix Riemann-Hilbert problem and the Schlesinger system.

We generalize our construction to derive integrable systems associated to arbitrary Hurwitz spaces $H_{g, N}$ of meromorphic functions of degree $N$ on Riemann surfaces of genus $g \geq 2$.

When the matrix dimension equals 1 , our systems are linear; they can be solved via scalar RiemannHilbert problem on the Riemann surfaces. Each solution of such system corresponds to a flat diagonal metric (Darboux-Egoroff metric), together with corresponding systems of hydrodynamic type and their solutions.

Our results suggest the following directions of future work. We expect our systems for $g \geq 2$ to be natural deformations of two-dimensional version of Hitchin systems proposed in [20]. The isomonodromic deformations in higher genus briefly discussed here should be in close relation to existing frameworks [24, 16, 19], as well as to non-autonomous Hitchin systems [1]. All these links should be clarified. The applications of the new systems should also be studied, especially from the point of view of their potential relationship with structures of Frobenius type.

Acknowledgments Our work on this paper was greatly influenced by Andrej Nikolaevich Tyurin. We thank M.Bertola, E.Ferapontov, T.Grava, and V.Sokolov for enlightening discussions. This research was supported by the grant of Natural Sciences and Engineering Research Council of Canada, grant of Fonds pour la Formation de Chercheurs et l'Aide a la Recherche de Quebec and the Faculty Research Development Program of Concordia University.

\section{References}

[1] Atiyah, M.F., Vector bundles over an elliptic curve, Proc.London Math. soc. (3) 7 414-452 (1957)

[2] Belinskii,V.A., Zakharov, V.E., Integration of the Einstein equations by the methods of inverse scattering theory and construction of explicit multisoliton solutions, Sov.Phys.JETP 48 (1978) 985-994

[3] Burtsev, S.P., Zakharov, V.E., Mikhailov, A.V., The inverse problem method with a variable spectral parameter, Teoret. Mat. Fiz. 70 no. 3 323-341 (1987)

[4] Courant, R., Hilbert, D., Methods of Mathematical Physics vol.2 New York: Interscience, 1962

[5] Dubrovin, B., Hamiltonian formalizm of Whitham-type hierarchies and topological LandauGinzburg models, Commun.Math.Phys., 145 195-207 (1992)

[6] Dubrovin, B., Geometry of 2D topological field theories, in: Integrable systems and quantum groups. Proceedings, Montecatini Terme, 1993, pp. 120-348, Lecture Notes in Math., v.1620, Berlin: Springer, 1996

[7] Dubrovin, B., Painlevé transendents in two-dimensional topological field theory, in The Painlevé property pp. 287-412, CRM Ser. Math. Phys. New York: Springer, 1999 
[8] Fay, J., Kernel functions, analytic torsion, and moduli spaces, Memoirs of the AMS, 96 No.464, AMS (1992)

[9] Fulton, W., Hurwitz schemes and irreducibility of moduli of algebraic curves, Ann. of Math., 90 542-575 (1969)

[10] J.Gibbons and S.P.Tsarev, Conformal maps and reductions of the Benney equations, Phys. lett. A, 258 (1999) 263.

[11] Hitchin, N., Stable bundles and integrable systems, Duke Math. Journ. 54 (1) 91-114 (1987)

[12] Jimbo, M., Miwa, M., Ueno, K., Monodromy preserving deformations of linear ordinary differential equations with rational coefficients, I, Phys. D 2 306-352 (1981)

[13] A.Kokotov, D.Korotkin, Some integrable systems on Hurwitz spaces, archive math-ph/0112051

[14] Korotkin, D., Finite-gap solutions of stationary axially symmetric Einstein equations in vacuum, Theor. Math.Phys., 77 No.1 1018-1031 (1989)

[15] Korotkin, D., Nicolai, H., Isomonodromic quantization of dimensionally reduced gravity, Nucl.Phys B 475 (1996) 397-439

[16] Korotkin, D., Samtleben, H., On the quantization of isomonodromic deformations on the torus, Int.J.Mod.Phys. A12 2013-2030 (1997)

[17] Krichever, I.M., Method of "averaging" for two-dimensional "integrable" equations, Funct. Anal. Appl. 22 no.3 200-213 (1989)

[18] Krichever, I.M., Spectral theory of two-dimensional periodic operators and its applications, Russ. Math. Surv. 44 (1989) 145-225.

[19] Krichever, I.M., Isomonodromy equations on algebraic curves, canonical transformations and Whitham equations, archive hep-th/0112096

[20] Krichever, I.M., Vector bundles and Lax equations on algebraic curves, Commun. Math.Phys., 229 229-269 (2002)

[21] Kupershmidt, B.A., Manin, Yu,I., Long wave equations with a free surface. I. Conservation laws and solutions., Functional Analysis and its applications, 11 No.3, 31-42 (1977)

[22] Maison, D., Are the stationary axially symmetric Einstein equations completely integrable? Phys.Rev.Lett. 41 (1978) 521-524

[23] Narasimhan, M.S., Seshadri, C.S., Stable and unitary bundles on a compact Riemann surface, Ann. of Math., 82 540-567 (1965)

[24] Levin, A.M., Olshanetsky, M.A., Hierarchies of isomonodromic deformations and Hitchin systems, archive hep-th/9709207

[25] Rauch, H.E., Weierstrass points, branch points, and moduli of Riemann surfaces, Comm. Pure Appl. Math. 12 543-560 (1959) 
[26] Tsarev, S.P., Geometry of hamiltonian systems of hydrodynamic type. Generalized hodograph method. Izvestija AN USSR Math. 54 No.5 1048-1068 (1990)

[27] Shramchenko, V., Integrable systems related to elliptic branched coverings, to appear

[28] Zverovich, E.I., Boundary value problems in the theory of analytic functions in Hölder classes on Riemann surfaces. Russ.Math.Surveys 26 no. 1 113-179 